\title{
Particles adsorbed at various non-aqueous liquid-liquid interfaces
}

Miguel Angel Fernandez-Rodriguez, ${ }^{,}{ }^{*}$ Bernard P. Binks, ${ }^{2}$ Miguel Angel Rodriguez-Valverde, ${ }^{1}$ Miguel Angel Cabrerizo-Vilchez ${ }^{1}$ and Roque Hidalgo-Alvarez ${ }^{1}$

${ }_{1}^{1}$ Biocolloid and Fluid Physics Group, Applied Physics Department, Faculty of Sciences, University of Granada, 18071-E Granada, Spain

${ }^{2}$ School of Mathematics and Physical Sciences, University of Hull, Hull. HU6 7RX. UK

*e-mail: mafernandez@ugr.es

Invited contribution to Dominique Langevin Festschrift on July 24th, 2017

Revised on 12.12 .16 


\begin{abstract}
Particles adsorbed at liquid interfaces are commonly used to stabilize water-oil Pickering emulsions and water-air foams. The fundamental understanding of the physics of particles adsorbed at water-air and water-oil interfaces is improving significantly due to novel techniques that enable the measurement of the contact angle of individual particles at a given interface. The case of non-aqueous interfaces and emulsions is less studied in the literature. Non-aqueous liquid-liquid interfaces in which water is replaced by other polar solvents have properties similar to those of water-oil interfaces. Nanocomposites of non-aqueous immiscible polymer blends containing inorganic particles at the interface are of great interest industrially and consequently more work has been devoted to them. By contrast, the behaviour of particles adsorbed at oil-oil interfaces in which both oils are immiscible and of low dielectric constant $(\varepsilon<3)$ is scarcely studied. Hydrophobic particles are required to stabilise these oil-oil emulsions due to their irreversible adsorption, high interfacial activity and elastic shell behaviour.
\end{abstract}

PACS:

$64.75 \mathrm{Xc}$

68.05.-n

$68.18 \mathrm{Fg}$

68.18-g,

68.47 Pe

77.84.Nh

82.70 Dd

82.70.Kj

Keywords: Oil-oil interfaces; Interfacial activity; Low dielectric constant; Particle-laden interfaces; Water-free emulsions. 


\section{Contents}

1. Introduction

2. Adsorption of particles at liquid interfaces: Theoretical aspects

3. Characterization of interfacial activity

4. Interfacial activity of particles adsorbed at non-aqueous liquid-liquid interfaces: Experimental data

4.1 Non-aqueous polar liquid-oil interface

4.2 Polymer-oil and Polymer-polymer interfaces

4.3 Oil-oil interfaces

5. Conclusions

Acknowledgements

References 


\section{Introduction}

The knowledge of the physicochemical aspects regarding the adsorption of particles at liquid interfaces is of great importance in understanding the behaviour of emulsions stabilised by particles [1,2]. Using particles instead of ionic or non-ionic surfactants present some advantages including the high adhesion energies that the particles possess at liquid interfaces and the Pickering effect that prevents coalescence when the particles covering an emulsion droplet come in contact [3]. Identifying the polar and apolar regions of molecular surfactants permits the prediction to some extent of their adsorption physics to different liquid interfaces [4]. This is less straightforward for particles, in which different parameters like the chemical nature, size, roughness and wettability play a role in the adsorption energy displayed by the particles at a given liquid interface $[5,6,7]$.

The vast majority of interfaces and emulsions stabilised by particles reported in the literature involve an aqueous phase and an oil phase. Nevertheless, here we will focus on the less studied case of non-aqueous liquid-liquid interfaces, and in particular, oil-oil interfaces [8]. Water-free or non-aqueous liquid-liquid interfaces are rather wide concepts covering interfaces in which the water phase in a water-oil system is replaced by either a polar non-aqueous solvent, a liquid polymer or another oil $[9,10]$. When the water phase is replaced by a polar solvent like propylene glycol [11], glycerol [12,13], formamide [14,15,13,16,17] or methanol [18], the solvent-oil interface is expected to retain the essential physics as that with the water phase [9]. On the other hand, the cases of liquid polymer-oil [19,20,21,22,23] and immiscible liquid polymer blends [24] $[25,26,27,28,29,30,31,32,33,34][35,36,37,38,39,40,41,42][43,44]$ are quite different and reported more frequently in the literature due to their important industrial applications. Nevertheless, we want to stress also the less studied case of true oil-oil interfaces. Such oils need to be largely immiscible but also need to possess a very low dielectric permittivity $\varepsilon$ (typically $<5$ ). This way, the oil-oil interface is one in which any polar interaction arising from the particles adsorbed at the interface will be significant. It is scarcely reported in the literature [9]. This is the principal motivation of this review: to compile the most recent works about particles adsorbed at a range of non-aqueous liquid-liquid interfaces, including the case of oil-oil interfaces.

\section{Adsorption of particles at liquid interfaces: Theoretical aspects}

Molecular surfactants, surface-active polymers and particles are used widely for stabilising emulsions and foams [45]. Nevertheless, although particles can display similar behaviour to molecular surfactants and surface-active polymers, they differ in two main aspects:

1) Particles do not assemble to form micelles (i.e. there are no solubilisation phenomena) [45].

2) Particles are solid-like entities incapable of the same spatial reconfiguration and bending of 
molecular surfactants and surface-active polymers. However, soft particles like microgels rearrange to some extent when placed at a liquid-liquid interfaces depending on their crosslinking density [46]. Such rearrangement is not as severe as the spatial reconfigurations that molecular surfactants and polymers experience however.

Usually, the contact angle $\theta$ or the flotation height h (they are related, see Figure 1a [47]) can be used to describe the attachment of a perfectly smooth spherical particle adsorbed at an interface. In the Young's equation 1 , the contact angle $\theta$ is related to the interfacial tensions involved in the attachment of the particle to the interface (see Figure 1a) [48]. Thus, $\gamma_{P f 2}$ (and $\gamma_{P f 1}$ ) are the interfacial tensions between the particle and the upper (and bottom) fluids and $\gamma_{\mathrm{f} 1 f 2}$ is the interfacial tension between the two fluids in the absence of particles.

$$
\gamma_{P f 2}-\gamma_{P f 1}-\gamma_{\mathrm{f} 1 \mathrm{f} 2} \cos \theta=0
$$

This equation brings two important approximations: the line tension and the roughness are neglected. The line tension $\tau$ depends on the three-phase contact line of the particle attached to the interface (see Figure 1a). For larger particles this line tension can be neglected, but for smaller nanoparticles the line tension can even cause detachment from the interface [49]. Moreover, the effect of the roughness of the particles cannot be neglected in the case of small nanoparticles [47].

If $\gamma_{P f 2}>\gamma_{P f 1}$, the particles prefer to be more immersed in fluid 1 (we would refer to them as hydrophilic if this is water) and they display contact angles in the range $\left(0-90^{\circ}\right)$, see Figure $1 \mathrm{~b}$. If the opposite occurs, $\gamma_{P f 2}<\gamma_{P f 1}$, the particles prefer to be more immersed in fluid 2 (we would refer to them as hydrophobic in a typical water-oil interface) and they display contact angles in the range (90-180 $)$, see Figure $1 b$.

If the line tension cannot be neglected, the Young's equation can be expressed as in equation 2 .

$$
\frac{\gamma_{P f 2}-\gamma_{P f 1}}{\cos \theta}-\gamma_{\mathrm{f} 1 \mathrm{f} 2}+\frac{\tau}{R \sin \theta}=0
$$

where $R$ is the particle radius. This equation still does not account for the roughness of the particle, which cannot be neglected for small nanoparticles. However, as this roughness cannot be expressed in an analytical way, the roughness effect needs to be incorporated for each experimental system. The simplest model of the energy of desorption ( $E_{\text {des }}$ ) of a particle attached at a given interface is given by equation 3 which is derived from Young's equation 1.

$$
E_{\text {des }}=\pi R^{2} \gamma_{\mathrm{f} 1 \mathrm{f} 2}(1 \pm \cos \theta)^{2}
$$


In equation 3, $\theta$ is the Young three-phase contact angle [45]. The sign inside the bracket is positive when the particle is more immersed in fluid 2 and negative when it is more immersed in fluid 1. Thus, even small particles of few nanometers in size can be irreversibly attached to interfaces provided that $\gamma_{f 1 f 2}$ and $\theta$ are adequate. However, the size of the particle is the main factor for a given interface because $E_{d e s}$ is proportional to the square of the particle radius.

We can consider the particles irreversibly attached to the interface when the energy of adsorption is greater than the thermal energy $k_{B} T$, where $k_{B}$ is the Boltzmann constant and $T$ the temperature measured in Kelvin [50]. To get an idea about the order of magnitude of the desorption energy of particles from interfaces, we will consider an example in which $\gamma_{f 1 f 2}=50 \mathrm{mN} / \mathrm{m}$, typical of an alkane-water interface. The value of $E_{d e s}$ of the particle depends then on its radius $\mathrm{R}$ and its contact angle $\theta$. As can be seen in Figure $1 \mathrm{c}, E_{\text {des }}$ depends on $\theta$ displaying a maximum and a minimum for the extreme cases of $\theta=0^{\circ}$ or $180^{\circ}$ respectively. For a particle attached to the interface with $\theta=90^{\circ}$, it can be seen that the energy of desorption is $\sim 0.4 k_{B} T$ for particles of $10 \mathrm{~nm}$ and $\sim 4,000 k_{B} T$ for particles of $1 \mu \mathrm{m}$. Thus, the major effect of particle size on the energy of desorption is clearly seen where bigger particles can be irreversibly attached compared with smaller nanoparticles.

If equation 2 is considered to include the line tension effects in the energy of desorption, equation 4 becomes a more complete and complex description of the energy of desorption, provided that the roughness effects cannot be analytically added to this expression [47].

$$
E_{\text {des }}=\gamma_{\mathrm{f} 1 \mathrm{f} 2} \cos \theta 2 \pi R^{2}\left(1-\cos \theta_{\mathrm{m}}\right)+2 \tau \pi R \sin \theta_{\mathrm{m}}-\pi R^{2} \gamma_{\mathrm{f} 1 \mathrm{f} 2}\left(\cos \theta_{\mathrm{m}}\right)^{2}
$$

In equation $4, \theta$ is the contact angle defined by the Young's equation 1 and $\theta_{m}$ is the experimentally accessible angle. Only if there is mechanical equilibrium, $\partial \mathrm{E}_{\mathrm{des}} / \partial \theta_{\mathrm{m}}=0$ and $\theta$ can be written as in equation 5 .

$$
\cos \theta_{\mathrm{m}}=\cos \theta\left(1-\frac{\tau}{R \gamma_{\mathrm{f} 1 \mathrm{f} 2}}\right)^{-1}
$$

This equation shows that for $\theta_{\mathrm{m}} \sim 90^{\circ}$ the contact angle $\theta$ is similar to that given in the simpler equation 1 because the line tension is perpendicular to the interface and the tangential component, which affects the immersion height of the particle, is negligible. Once again, the maximum contribution of the line tension is for the extreme cases of $\theta_{\mathrm{m}}=0^{\circ}$ and $180^{\circ}$, while it is not relevant when the contact angle is between $60^{\circ}$ and $120^{\circ}$ [51].

All the above expressions are obtained for homogeneous particles with a homogeneous surface. One way to improve the amphiphilic character of particles at interfaces is to functionalise the particle surface with capping ligands [52,53,54]. Thus, the particle can be functionalised with two 
capping ligands which are better solvated in one of the two fluids of the interface. Typically, for a water-oil interface, such capping ligands are hydrophilic and hydrophobic to enhance their solvation in water and oil, respectively. The capping ligands can be arranged at the particle surface totally mixed, forming patches or in a dissymmetric Janus configuration. The latter Janus configuration leads to an enhanced interfacial activity of the nanoparticles provided that the two capping ligands, hydrophilic and hydrophobic, display affinities for the water and oil fluids, respectively [55]. Thus, the Janus particle is not only surface-active but also amphiphilic just like molecular surfactants are that present a hydrophilic part and a hydrophobic part. For example, homogeneous particles with a contact angle of $90^{\circ}$ are strongly surface-active although they are not amphiphilic. Instead, Janus particles are both surface-active and amphiphilic [55]. The amphiphilicity of Janus particles at a water-oil interface can be tuned through variation of both the angle $\alpha$ (relative areas of the polar and apolar domains, see Figure 2) and the difference between $\theta_{\mathrm{A}}$ and $\theta_{\mathrm{P}}$ (equilibrium contact angles of the two domains). The homogeneous particles show zero amphiphilicity $\left(\alpha=0^{\circ} / 180^{\circ}\right.$ or $\left.\left(\theta_{\mathrm{A}}-\theta_{\mathrm{P}}\right)=0^{\circ}\right)$. Janus particles show the strongest amphiphilicity when $\alpha=90^{\circ}$ and $\left|\theta_{\mathrm{A}^{-}} \theta_{\mathrm{P}}\right|=180^{\circ}$.

The surface free energy $E$ of a Janus particle at a water-oil interface as a function of the immersion angle $\beta$ (see Figure 2) is given by equations 6 and 7 [55].

For $\beta \leq \alpha$,

$$
E(\beta)=2 \pi R^{2}\left[\gamma_{A O}(1+\cos \alpha)+\gamma_{P O}(\cos \beta-\cos \alpha)+\gamma_{P W}(1-\cos \beta)-\frac{1}{2} \gamma_{O W}\left(\sin ^{2} \beta\right)\right]
$$

and for $\beta \geq \alpha$,

$$
E(\beta)=2 \pi R^{2}\left[\gamma_{A O}(1+\cos \beta)+\gamma_{A W}(\cos \alpha-\cos \beta)+\gamma_{P W}(1-\cos \alpha)-\frac{1}{2} \gamma_{O W}\left(\sin ^{2} \beta\right)\right]
$$

In equations 6 and 7, $\gamma_{\mathrm{AO}}, \gamma_{\mathrm{PO}}, \gamma_{\mathrm{AW}}, \gamma_{\mathrm{PW}}$ and $\gamma_{\mathrm{OW}}$ are the interfacial tensions of the apolar-oil, polar-oil, apolar-water, polar-water and oil-water interfaces, respectively. Equations 6 and 7 neglect the radius of curvature of the oil-water interface relative to the particle radius, the line tension and the buoyancy effects and consider that the apolar (polar) region is oriented towards the apolar (polar) fluid. Thus, the energy of desorption still scales with $\gamma o w R^{2}$ and all the casuistry explained for homogeneous particles apply for Janus particles. The contact angles $\theta_{\mathrm{A}}$ and $\theta_{\mathrm{P}}$ correspond to the equilibrium angles given by the Young's equation 1 for the apolar and polar part of the particle given in equations 8 and 9 [55].

$$
\begin{aligned}
& \cos \theta_{A}=\frac{\gamma_{A W}-\gamma_{A O}}{\gamma_{O W}} \\
& \cos \theta_{P}=\frac{\gamma_{P W}-\gamma_{P O}}{\gamma_{O W}}
\end{aligned}
$$


Thus, an average contact angle can be obtained by weighting the relative areas of the polar and apolar domains as in equation 10 [55].

$$
\theta_{\text {average }}=\frac{\theta_{A}(1+\cos \theta)+\theta_{P}(1-\cos \alpha)}{2}
$$

The three possible immersion angles $\beta$ are listed in equation 11 [55].

$$
\begin{aligned}
& \text { For } \alpha<\theta_{\mathrm{A}}<\theta_{\mathrm{P}} \text {, then } \beta=\theta_{\mathrm{A}} \\
& \text { For } \theta_{\mathrm{A}}<\alpha<\theta_{\mathrm{P}} \text {, then } \beta=\alpha \\
& \text { For } \theta_{\mathrm{A}}<\theta_{\mathrm{P}}<\alpha \text {, then } \beta=\theta_{\mathrm{P}}
\end{aligned}
$$

The energy of desorption can be calculated from equations 6, 7 and 11. Figure 3 shows the energy of desorption for particles with different values of the angle $\Delta \theta$ (defined as $\left|\theta_{\mathrm{A}^{-}} \theta_{\mathrm{P}}\right| / 2$ ). In Figure $3, \alpha=$ $90^{\circ}, \mathrm{r}=10 \mathrm{~nm}$ and $\gamma_{0 w}=36 \mathrm{mN} \mathrm{m}^{-1}$. Thus, the particle amphiphilicity is changed by $\Delta \theta$. The extreme cases of $\Delta \theta=0^{\circ}$ and $180^{\circ}$ correspond to the homogeneous hydrophilic/hydrophobic nanoparticles and $\Delta \theta=90^{\circ}$ corresponds to a Janus particle in which the polar region of the particle is completely immersed in the polar fluid and the apolar region is completely immersed in the apolar fluid. As can be seen in Figure 3, by increasing the particle amphiphilicity through $\Delta \theta$, the strength of particle adsorption increases up to a maximum of 3 -fold for $\theta_{\text {average }}$ of $90^{\circ}$. In addition, the Janus particles maintain strong adsorption with average contact angles near $0^{\circ}$ or $180^{\circ}$, where the surface activity of the homogeneous particles is low. While this discussion is done in terms of apolar and polar regions, these results might be extended for oil-oil interfaces by considering that the polar and apolar fluids are instead the two more dense and less dense apolar fluids. In such a situation, the apolar affinity of the two capping ligands might be tuned through their hydrophobicity, provided that the two oils possess a difference in hydrophobicity to some extent.

Moreover, van der Waals interactions and electrostatic interactions between interfacial particles become even more important when there is not a polar phase in which a screening effect of the particle charge can occur. van der Waals interactions arise from the orientation-averaged interactions between permanent dipoles (Keesom interactions), interactions of permanent dipoles with induced dipoles (Debye interactions) and interactions between fluctuating dipoles (London interactions). The first two interactions occur only for permanent dipoles, while the London interaction is always present and it is always attractive for two like particles in a given medium [52]. Steric repulsive effects are also important when the particles are functionalised with polymers since they exert an osmotic pressure when compressed between two particles [52]. Finally, capillary interactions are usually neglected for smaller nanoparticles because they originate from deformations of the fluid-fluid interface. Such deformation usually comes from the weight of the particle which is 
negligible for sub-micron particles. However, even for small nanoparticles, the roughness of the nanoparticle surface may lead to deformation of the interface due to pinning effects $[52,56,57,58]$, which makes the capillary interactions significant.

\section{Characterization of interfacial activity}

The usual way to characterise the interfacial activity of particles adsorbed at interfaces is to measure the interfacial pressure, being the interfacial tension of the bare interface minus that of the particle covered interface. This can be done by several methods including using a Langmuir balance or via pendant drop tensiometry amongst others [50]. While the Langmuir balance is less complex than pendant drop tensiometry because it measures the interfacial pressure directly with a Wilhelmy plate and compresses the interface by moving barriers, pendant drop tensiometry allows using significantly lower volumes of the fluids and lower quantities of particles. This is especially useful with particles that cannot be synthesised at laboratory scale in large quantities as is the case for most Janus nanoparticles [50]. Pendant drop tensiometry is composed of a CMOS (complementary metal-oxide semiconductor image sensor) camera and a computer, which performs the acquisition of images. Then, the drop shape is fitted with edge-detecting software to the Young-Laplace equation using the Axisymmetric Drop Shape Analysis Profile (ADSA-P). Real time drop images are processed at each step of volume variation and the drop area and interfacial tension are calculated. This system can be used in cases where the interfacial tension is low, as expected for oil-oil interfaces [59]. An entropic edge detector has been applied to a pendant drop of the aqueous phase in the oil phase obtained after mixing 1-propanol, n-heptane and water. In this experiment, a pendant drop not bigger than $0.32 \mu \mathrm{L}$ is formed with very low contrast of the images (see Figure 4). In these conditions, only the entropic edge detector is useful and low interfacial tensions can be measured at different temperatures (see Figure 5). The interfacial tension decreases upon increasing temperature, from $0.262 \mathrm{mN} / \mathrm{m}$ at $18{ }^{\circ} \mathrm{C}$ to 0.080 $\mathrm{mN} / \mathrm{m}$ at $70{ }^{\circ} \mathrm{C}$. This method is also demonstrated in this review for oil-oil interfaces containing attached particles with new results measured in our lab. in Section 4.3.

As seen in the previous Section 2, the macroscopic interfacial tension is related to the microscopic contact angle (i.e. immersion depth) of the particles at a given interface. In principle, determining this microscopic contact angle should permit one to estimate the interfacial activity of the particles at the interface. However, this microscopic contact angle is difficult to measure and it is even very dependent on the experimental technique [47]. One common assumption is that the contact angle for particles at an interface is the same as that for a flat macroscopic surface made of the same material or from close-packed monolayers of particles deposited onto a flat substrate. This approach neglects microscopic effects as roughness, line tension and even thermal fluctuations [47]. Another assumption is that the contact angle can be obtained from measurement of the collapse pressure 
which corresponds to a close-packed monolayer. This is not the case however for many systems in which there may not be collapse or this might occur for non close-packed percolating colloidal layers (in particular with non-homogeneous particles as Janus particles) [50]. There are relaxation processes such as buckling that can modify the contact angle of particles at the interface under compression [39].

Other techniques are based on the immobilization of the particles at interfaces to directly measure their contact angle as with the Gel Trapping Technique (in which the aqueous phase is gelled) [60] or the Freeze-Fracture Shadow-Casting (FreSCa) Cryo-SEM technique (in which the interface is vitrified and fractured, shadow casting the particles at the interface with tungsten) $[61,62]$. However, as mentioned before, such techniques provide different contact angles even for the same technique by varying parameters like the spreading solvent or slightly changing the size of the particles [47]. For example, $2.8 \mu \mathrm{m}$ polystyrene particles at a water- decane interface exhibit a contact angle of $85^{\circ}$ measured by FreSCa and $122^{\circ}$ measured by the Gel Trapping Technique [61]. Nonetheless, the higher contact angles measured by the latter were explained in terms of the most hydrophilic particles remaining embedded in the gellan gum after the PDMS replica was peeled off and hence were 'missing' in the final contact angle distribution [63]. Very recently, it was shown that attaching a microparticle to an AFM cantilever enables the contact angle to be determined via detachment of the particle from the interface [63].

These techniques can be useful to relate the microscopic and macroscopic behaviour. For example, it can be seen how the microscopic aggregation state of PMMA homogeneous nanoparticles (PMMA-HPs) and silica nanoparticles functionalised with methacryloxypropyltrimethoxysilane (silica-FPs) at the water-decane interface (see Figure 6) is related with the macroscopic appearance (see Figure 7). The interface is clear for the well dispersed PMMA-HPs but it is turbid for the aggregated silica-FPs [62]; in the latter, particles aggregates are visible at the interface at the back of the pendant drop (out of focus). Moreover, further evidence that they are placed at the interface is that when there is a drift all the particles move together like a shell around the interface. These techniques cannot be employed in non-equilibrium conditions. All of them need the nanoparticles to be immobilised in one way or another. However, there are more complex techniques which enable one to measure the interfacial activity of particles at interfaces in non-equilibrium conditions. For example, neutron reflectivity allows in-situ measurements of the contact angle of nanoparticles adsorbed at fluid interfaces (see Figure 8) [64]. This technique was used to measure two sets of 4.8 nm gold nanoparticles: homogeneous particles coated by perdeuterated 1-octanethiol (d-OT) and functionalised particles coated by perdeuterated 1-octanethiol and 6-mercapto-1-hexanol (d-OT:MHol 1:1). The contact angle of the former particles was $119.5 \pm 5.5^{\circ}$, in good agreement with simulations. The functionalised particles displayed a contact angle of $85 \pm 10^{\circ}$, much lower than that of d-OT particles as expected due to the presence of hydrophilic ligands. With this technique it was even 
possible to determine the structure of the capping ligand on the functionalised particles, showing a random mixing of the two capping ligands as opposed to a Janus dissymmetric structure [64]. Moreover, modern techniques as ultrafast three-dimensional imaging upon irradiation with light, Xrays or electrons enable to find in real time the orientation and position of individual nanoparticles at interfaces $[65,66,67]$. These techniques however require significantly larger effort in the data extraction but might be a good route in the future to characterise the interfacial activity of nanoparticles in real-time non-equilibrium conditions.

In order to obtain the rheological characteristics of the interfaces with adsorbed particles there are two main paths: bulk 3D-rheology and 2D-interfacial dilatational rheology. The bulk 3D-rheology in which an emulsion prepared with the two fluids and the particles is subjected to periodic shear or squeeze deformations obtain the 3D viscosity and elasticity of the emulsion as a function of the shear or squeeze rate and time. This bulk characterization provides important information concerning the flow behaviour and the stability of the emulsions, useful for practical applications [20]. Moreover, this can be performed at the same time as the real structure of the emulsion is obtained by confocal microscopy [25]. Nevertheless, this bulk characterization might not capture all the physics undergoing at the 2D-interface level. The interfacial dilatational rheology can be measured to obtain the corresponding interfacial viscosity $\eta_{\mathrm{d}}$ and elasticity $E_{d}$ by increasing and decreasing periodically the area of the interface with adsorbed particles. This is achieved by compressing and expanding the interface confined within the barriers of a Langmuir balance or by growing and shrinking a pendant drop of one fluid immersed in a second fluid [50].

\section{Interfacial activity of particles adsorbed at non-aqueous liquid-liquid interfaces: Experimental data}

Binks and Tyowua [9] recently reported an extensive compendium of the different experimental works, including patents, of the behaviour of non-aqueous liquid-liquid interfaces with adsorbed particles. They reported that it was necessary to distinguish between three cases: (i) substitution of the water phase in a water-oil mixture by a polar solvent of high dielectric constant, (ii) blends of immiscible liquid polymers and (iii) mixtures of two immiscible oils of low dielectric constant $(\varepsilon<$ 3.2). In addition, oil-air interfaces stabilised by either fatty alcohol [68] or fatty acid [69] crystals have been reported to be very stable over time and they have applications in diverse fields such as food and cosmetic industries. In any case, the phrase oil-oil is a matter of discussion because "oil" is usually used to denote substances that are not hydrophilic and in a more generic way the Encyclopaedia Britannica says that an oil is "any greasy substance that is liquid at room temperature and insoluble in water". After all, the term "oil" is not compounded in the IUPAC Golden Book [70]. This loose concept 
explains why some authors name polar-non-polar interfaces or polymer blends as oil-oil interfaces [9].

Although there is a plethora of different particles and different types of non-aqueous liquidliquid interfaces reported in the literature, the vast majority belong to the second category of polymer blends as will be noted in the following sub-sections. We take the example of microgel particles, which are polymeric particles with a more cross-linked core and a less cross-linked corona. These are of interest due to their capability of being temperature or pH-sensitive, typically becoming swollen below or above a given value of temperature or $\mathrm{pH}$ [71]. Microgels are excellent colloidal probes at water-air and water-oil interfaces. At the water-hexane interface, they are reported to produce colloidal monolayers beyond the close-packing regime [71]. These microgels have been well characterised at water-hexane interfaces and observed to be shaped as fried eggs due to the poor solvation of the particular microgel in the oil phase [72]. Even though these results involve the use of water and microgels, which are well dispersed in water, there are also non-aqueous microgels reported in the literature which are expected to retain the same physics fundamentals at non-aqueous liquid interfaces [73]. For such non-aqueous microgels, the main factor determining the colloidal stability in oil is the steric barrier afforded by polymer chains extending into solution. Moreover, microgels are reported to be capable of stabilising non-aqueous emulsions [14]. In the following subsections, we compile the main experimental data of particles adsorbed at non-aqueous liquid-liquid interfaces.

\subsection{Non-aqueous polar liquid-oil interface}

If the water in a water-oil emulsion is replaced by a polar solvent, the emulsion is expected to retain the same fundamental behaviour, although it might be comparatively less polar than water-containing emulsions (since water has a dielectric constant $\varepsilon$ of $\sim 80$ at $20^{\circ} \mathrm{C}$ ). Binks et al. [11] prepared emulsions of propylene glycol $(\varepsilon \sim 32)$ and paraffin $(\varepsilon \sim 2)$ stabilised by fumed silica particles of different hydrophilicity. They reported on the appearance and stability of the emulsions as a function of the particle hydrophilicity (see Figure 9). It was necessary to lower the hydrophilicity of the particles (i.e. reduce the silanol content at particle surfaces) to obtain transitional phase inversion compared to using water as the polar solvent. Thus, the particles behave as more hydrophilic in propylene glycol systems than in water. The glycol was not completely emulsified for particles forming glycol-in-oil emulsions (14\% SiOH). For oil-in-glycol emulsions, even after 6 months, the emulsions were stable against coalescence and creaming for the more hydrophobic particles. For 61\% and $71 \% \mathrm{SiOH}$, coalescence and creaming occurred, and above this percentage complete phase separation occurred immediately after emulsion formation (see Figure 9). Thus, Ostwald ripening needs also to be taken into consideration [2] this does not follow on. They reported also that the 
emulsion average drop diameter was minimum at phase inversion (Figure 10(a)) with particularly small oil drops of around $5 \mu \mathrm{m}$ being stabilised by particles possessing $23 \% \mathrm{SiOH}$. The optical micrographs in Figure 10(b) revealed non-spherical drops for lower \% SiOH.

Dyab and co-workers [12], [13], [14] also investigated non-aqueous emulsions in which water was replaced by either glycerol $(\varepsilon>48)$, formamide $(\varepsilon \sim 109)$ or ethylene glycol $(\varepsilon \sim 37)$. These emulsions were stabilised by hydrophobised amorphous silica and organo-modified Laponite clay nanoparticles [12], kaolininte clay [13] or non-aqueous microgels [14]. Dyab and Atta [14] used crosslinked poly(N-isopropylacrylamide-co-2-acrylamido-2-methylpropane sulfonic acid), poly(NIPAM-coAMPS), microgels of diameter $600 \mathrm{~nm}$ functionalised with a non-ionic polymerisable surfactant (polyoxyethylene 4-nonyl-2-propyl-phenyl maleate ester). They measured the formamide-paraffin oil interfacial tension as a function of the microgel concentration in formamide. While the bare interface exhibited a tension of $29.5 \pm 0.9 \mathrm{mN} / \mathrm{m}$, they reported a significant reduction of the interfacial tension to around $3.5 \mathrm{mN} / \mathrm{m}$ by $2.5 \mathrm{wt} . \%$ of particles (see Figure 11) with equilibrium occurring quickly. Therefore, microgels adsorb at the formamide-paraffin interface as they do at the water-hexane interface [71]. They attributed the high surface activity to either the presence of charges on the AMPS monomer or to the formation of a 3-D network of particles in the continuous phase which can improve the stability of the emulsions. They also reported that unlike the water-based microgels studied by them earlier, these particular microgels did not show any sensitivity to temperature within the range of $20-80^{\circ} \mathrm{C}$. Moreover, Tawfeek et al. [13] explored the synergism between a polymerisable non-ionic surfactant (Noigen RN10) and kaolinite clay particles in stabilising these non-aqueous emulsions. Using kaolinite particles with equal volumes of paraffin oil and formamide resulted in no stable emulsions at all concentrations, although stability was enhanced by addition of Noigen RN10. They also reported that the addition of Noigen RN10 surfactant to stable silicone oil $(\varepsilon \sim 3)$-inglycerol emulsions containing kaolinite particles resulted in emulsion destabilization at all concentrations. Thus, the surfactant can improve or decrease the emulsion stability depending on the type of oil and hence on the nature of the polar liquid-oil interface. There was no evidence of catastrophic phase inversion in these emulsions (by variation of the formamide:oil ratio) as commonly occurs in water-oil Pickering emulsions. Additionally, Dyab and Al-Haque [12] reported on the preparation of emulsions, foams, liquid marbles and polymeric materials from a range of nonaqueous systems stabilised solely by either dichlorodimethylsilane (DCDMS)-modified amorphous silica or organo-modified Laponite clay nanoparticles, including examples of double emulsions like formamide-in-styrene $(\varepsilon \sim 2)$-in-formamide.

Datta et al. [15] also prepared emulsions of silicone oil-in-formamide but stabilised by Pluronic P105, a non-ionic amphiphilic copolymer, instead of particles. However, they measured in a systematic way the bulk rheology of those emulsions, concluding that emulsions exhibiting attractive interactions between drops show a dramatically enhanced elasticity compared to the usual repulsive 
emulsions. They found that the linear and non-linear rheology of such emulsions depended sensitively on the interactions between the droplets, proving useful to design emulsions with a specific elastic and flow behaviour. Rizelli et al. [18] prepared non-aqueous Pickering emulsions using anisotropic block copolymer nanoparticles. The emulsions were prepared with sunflower oil $(\varepsilon \sim 3)$, methanol $(\varepsilon$ 33) and poly(2-(dimethylamino)ethylmethacrylate)-poly(benzyl methacrylate) (PDMA-PBzMA) worm-like particles. They reported that emulsions prepared with increasing sunflower oil content were stable up to a volume fraction of 0.6. There was an ageing effect in all cases, in which the sunflower oil droplets gradually increased in size over time during several days. Nevertheless, after this period, the emulsions remained stable for at least 2 months at room temperature. As an example of the food industry interest in non-aqueous emulsions, Hu et al. [74] studied non-aqueous emulsions formed by a mixture of propylene glycol and Transcutol ${ }^{\circledR}$ CG (an ethoxydiglycol, $\varepsilon \sim 12$ ) and evening primrose seed oil. They named this kind of emulsion as "non-aqueous self-double-emulsifying drug delivery systems (SDEDDS)" and concluded that SDEDDS can spontaneously emulsify to 'oil'-in-'oil'in-water double emulsions with drugs encapsulated in the internal 'oil' phase. The SDEDDS were stable for up to 3 months at $40^{\circ} \mathrm{C}$.

\subsection{Polymer-oil and polymer-polymer interfaces}

Regarding the polymer-oil interface, Atanase and Riess [19,20,21] addressed in three different studies the interface formed by PEG 400 (polyethyleneglycol, $\varepsilon \sim 14$ ) and paraffin oil or Miglyol 812 (triglyceride, $\varepsilon \sim 4$ ) and stabilised by different block copolymers. They reported that the block copolymers formed reverse micelles in paraffin oil. The emulsion characteristics, such as quiescent stability, droplet size and rheological behaviour, were a function of the copolymer concentration and they were correlated with the copolymer molecular characteristics. They could even prepare biocompatible emulsions for topical applications [21]. However, the block copolymers are rather large surfactants with two differentiated regions compared to particles. Similarly, Voigt et al. [22] reported emulsion formation of a solution of poly(lactic-co-glycolic acid) in dimethyl sulphoxide $(\varepsilon \sim 47)$ dispersed in several vegetable oils. The stability of these emulsions was improved from a few min to $12 \mathrm{~h}$ through the addition of glycerol monostearate (GMS) to the continuous oil phase, providing a viscosity increase and the formation of a GMS layer at the emulsion drop interface. Also, the injectability of such emulsions was improved, allowing for a faster parenteral administration and hence a reduction of the pain exposure time for patients. Thus, the polymer-oil interfaces display useful biocompatible applications including drug delivery.

As stated before, there are plenty of studies devoted to polymer-polymer interfaces. The studies of immiscible polymer blends usually focus on the 3-D bulk rheology of emulsions stabilised by particles for their industrial applications and also usually involve high temperatures at which the 
polymers melt. Bai et al. [25] reported a non-polar bijel composed of styrene trimer and low molecular weight polybutene that was stabilised by hydrophobic silica nanoparticles (see Figure 12). They found that the silica nanoparticles at the interface suppressed coarsening in the bijels. Interfacial localization of the particles was demonstrated using confocal microscopy and cryo-SEM. Causa et al. [26] studied the morphology and texture of poly( $\varepsilon$-caprolactone)-polyethylene oxide blend films stabilised by titanium dioxide particles, hydroxyapatite particles and aluminium-magnesium layered double hydroxide platelets. They concluded that, as in the case of bulk multiphase systems, the morphology of the polymer blend could be controlled by either the blend composition or by the addition of small amounts of inorganic nanoparticles. Laoutid et al. [27] reported blends of polyamide- 6 and polycarbonate with alumina nanoparticles. The thermal and rheological measurements showed that the nanoparticles acted as protective agents reducing the thermal degradation of the polymer pair during melt processing. Liu et al. [28] studied blends of styrenebutadiene rubber and polyisoprene with organo-montmorillonite clay particles. They studied the effects of the organoclay particles on the phase separation behaviour and the morphology of the polymer blend by rheological methods. They found that even a small amount of clay particles could strongly influence the phase separation behaviour and the morphology evolution of the immiscible blends, raising their viscoelasticity and slowing down the phase separation. Maani and Carreau [29] reported the rheological and morphological behaviour of blends of polypropylene and ethyleneoctene copolymer containing nanosilica particles. The presence of nanosilica particles improved the morphological stability of all blends, but the effect was higher when the nanoparticles were localised in the dispersed ethylene- octene phase. While shearing diminished the viscoelastic properties of the blends in the absence of silica particles, the sheared nanocomposites (i.e. polymer blend with particles) enhanced the viscoelasticity due to the interconnection of solid particles during shearing. Mao et al. [30,31] reported two studies of blends of polyisobutylene and polydimethylsiloxane in the presence of either spherical or ellipsoid polystyrene particles. They concluded that during shearing, droplets became elongated and rotated periodically about their major axes while aligning along the vorticity direction in ellipsoid-filled emulsions due to both an extremely small Reynolds number that arrested the coalescence and a strong confinement effect. No such behaviour was observed in emulsions containing spherical particles however. Moghimi et al. [32] studied the effect of micronsized hydrophobic calcium carbonate particles on the stabilization of polydimethylsiloxane and polyisobutylene immiscible blends. The particles provided negligible contribution to the bulk rheology of the phases but suppressed the coalescence. Surface coverage calculations revealed that the steric barrier was not the stabilising mechanism because the droplet surface was scarcely covered by particles. They concluded that particle-induced droplet bridging might be the stabilization mechanism due to patchy interactions induced by the heterogeneous distribution of particles along the interface. Pawar and Bose [33] discussed the influence of the location of nanoparticles on the 
morphologies appearing in polymer blends. The increased yield stress of the particle-loaded phase slows down the relaxation resulting in arresting peculiar morphologies which would otherwise be thermodynamically unfavourable due to the increased interfacial area. In the case of interfacially adsorbed particles however, the resulting solid-like interfaces can also preserve the irregular structures. These transitions are very different to those in classical copolymer compatibilised polymer blends. Qian et al. [34] reported the morphology and crystallization behaviour of poly(Ecaprolactone) in its $80 / 20$ blends with poly(styrene-co-acrylonitrile) containing hydrophobic or hydrophilic nanosilica particles. It was found that hydrophilic nanosilica displayed a more significant effect in the morphology of the blends than hydrophobic nanosilica. Salehiyan et al. [36,37] studied polypropylene-polystyrene blends with either silica or clay particles. They proposed the normalised non-linear/normalised linear viscoelastic ratio (NLR) to determine the nature of the relation between droplet size and rheological properties. They found that the NLR and droplet size were inversely proportional, resulting in a promising tool to investigate the microstructural changes of polymer blends. Sangroniz et al. [35] reported the linear and non-linear rheological behaviour of polypropylene-polyamide blends with hydrophobic nanosilica particles. The size of the polyamide droplets in the emulsion was reduced up to 25 times when the particles were added. Trifkovic et al. [38] found that montmorillonite clay nanoparticles stabilised polymeric blends of polyethylenepoly(ethylene oxide). Thus, organically modified clays localised at the interface and provided complete suppression of coarsening, even at concentrations as low as 1 wt.\%. Zou et al. [39] studied the polybutadiene/polydimethylsiloxane (10/90) blend and the inverse system with fumed silica particles. The nanoparticles significantly affected the morphology of the blends, inducing droplet clustering and decreasing the droplet size, regardless of which phase preferentially wetted the particles. This was unexpected given that these particles usually displayed this behaviour when they were preferentially wetted by the continuous phase.

Fenouillot et al. [40] reported in a review that the reason for the uneven distribution of nanoparticles in polymer blends is linked to the wettability of the particles by the polymers. However, they mentioned the lack of reliable techniques to accurately determine the particle-polymer interfacial energy, especially at high temperatures. Moreover, Taguet et al. [41] in a review concluded that the nanoparticles have a great influence on the mechanical, barrier, thermal and fire properties of polymer blends mainly because of their size. The small size of the nanoparticles generates high interfacial area with the polymer chains such that their configurational entropy at the surface of nanoparticles is greatly decreased. As Salzano de Luna and Filippone [42] stated in a recent review: "imparting new physical properties and novel behaviour to a polymer blend through the simple addition of nanoparticles is what really makes polymer nanocomposites attractive". All of the above results for polymer blend nanocomposites are a selection of the large amount of work being devoted currently to polymer blends which deserves a review in itself. Here, they are contemplated as 
examples of non-aqueous emulsions.

\subsection{Oil-oil interfaces}

Oil-oil liquid interfaces are quite different from water-oil or polar solvent-oil interfaces, not only because of the low dielectric constant of both oils compared to polar solvents but also because their interfacial tension is particularly low $(<3-5 \mathrm{mN} / \mathrm{m})$. Moreover, the large chains within polymers compared to low molar mass oil molecules make their treatment different. For example, many oils are liquid at room temperature whereas many polymers usually need high temperatures to be melted.

Binks and Tyowua [9] recently published an extensive compilation of the studies concerned with oil-oil interfaces in the literature, including patents (see Table 1), and found that oil-in-oil emulsions are used in a wide variety of industries including cosmetics $[75,76,77]$, personal care $[78,79,75,80]$, electronics $[81,82,83]$ and pharmaceuticals. [84,85,86]. They are also used in antifoaming applications [87,88], in liquid toning [89] and as reaction vehicles involving reactants sensitive or explosive to the presence of traces of water [90]. However, only four studies listed in Table 1 used particles to stabilise the emulsions as opposed to molecular surfactants or polymers: the particle types were organo-clay [78], fluorosilicone [76], fluorolauroyl taurate [77] and wax [88]. Thus, a detailed understanding of particles adsorbed at oil-oil interfaces is still lacking. Therefore, they explored systematically the behaviour of immiscible mixtures of vegetable oil and silicone oil in the presence of fumed silica particles, coated with either hydrocarbon groups or fluorocarbon chains, and other particles such as fluorinated clay microplatelets. First, they measured the interfacial tension and miscibility of each pair of oils: sunflower, rapeseed and olive oil as one of the phases and PDMS silicone oil of different viscosities (20,50 and $100 \mathrm{cS})$ as the other phase. The vegetable oils were completely immiscible with the silicone oils. For all vegetable oil-silicone oil combinations, the interfacial tension was below $3 \mathrm{mN} / \mathrm{m}$. Taking the example of sunflower oil-20 cS PDMS silicone oil, the hydrophilic fumed silica particles (possessing 100\% SiOH on their surfaces) displayed an advancing and receding contact angle (through PDMS) of $150^{\circ}$ and produced an unstable silicone oilin-vegetable oil emulsion. On the other hand, the hydrophobic fumed silica particles (functionalised with DCDMS and with $14 \% \mathrm{SiOH}$ ) displayed an advancing and receding contact angle of $67^{\circ}$ and $44^{\circ}$, respectively, forming stable vegetable oil-in-silicone oil emulsions.

The behaviour of oil-oil emulsions was studied in a systematic way. Emulsions of equal volumes of sunflower oil or olive oil and 20 cS PDMS silicone oil and 1 wt.\% of DCDMS-coated silica particles with different $\% \mathrm{SiOH}$ on their surfaces were prepared. The particles were not pre-dispersed in either oil, but added as a powder. The appearance of the emulsions after one month is shown in Figure 13. The most hydrophilic particles $(100 \% \mathrm{SiOH})$ and particles of intermediate hydrophobicity (88-25\% SiOH) formed silicone oil-in-vegetable oil emulsions which were extremely unstable to coalescence with complete phase separation within several days. For more hydrophobic particles 
(23\%), stable silicone oil-in-vegetable oil emulsions were formed. For the most hydrophobic particles possessing $14 \% \mathrm{SiOH}$ for sunflower oil or either $20 \%$ or $14 \% \mathrm{SiOH}$ for olive oil, stable vegetable oil-insilicone oil emulsions were obtained. The authors concluded that the most stable emulsions to creaming and coalescence were obtained with the most hydrophobic particles [9].

Moreover, Binks and Tyowua [9] explored this behaviour with other hydrophobic particles like PF-5 Eight Pearl 300S-Al powder, which contains partially fluorinated sericite clay platelet microparticles. They studied the mixture of sunflower oil-20 cS silicone oil at different clay platelet concentrations (see Figure 14(a)). The emulsions were vegetable oil-in-silicone oil and the extent of creaming and coalescence decreased with increasing particle concentration (see Figure 14(b)), where it can be seen that particle concentrations > $1 \mathrm{wt}$ \% produced emulsions stable to coalescence. Moreover, the cryo-SEM image in Figure 14(c) showed the vegetable oil as rough compared with the smooth silicone oil. The spherical silicone particles in this case were positioned at the interface of the two oils and are relatively close-packed. These findings reinforced the results with silica particles highlighting that hydrophobic particles of different size and shape were effective stabilisers of oil-inoil emulsions.

We have characterised the interfacial activity of the fumed silica hydrophobic particles (14\% $\mathrm{SiOH}$ ) and PF-5 Eight Pearl 300S-Al particles used in the study of Binks and Tyowua [9] by pendant drop tensiometry, which proved successful in low interfacial tension systems [59]. We dispersed different concentrations of particles in 20 cS PDMS silicone oil and formed a pendant drop in air. Next, the pendant drop (which may have adsorbed particles) was immersed in sunflower oil (purified by mixing with florisil salt and further filtration to remove polar impurities). The interfacial activity and interfacial dilatational rheology was obtained as in previous work [54]. First, the oil-oil interfacial tension was monitored for a $40 \mu \mathrm{L}$ pendant drop until it was stable (5 min for the silica particles and $80 \mathrm{~min}$ for the clay particles). Next, the interfacial tension was monitored during the exchange of the silicone oil drop phase, achieved with a double capillary and two micro-injectors to see if the particles were irreversibly attached at the interface when the drop phase was renewed with fresh 20 cS PDMS silicone oil. Moreover, the interfacial activity was also characterised by growing and shrinking cycles (between 40 and $5 \mu \mathrm{L}$ at $0.1 \mu \mathrm{L} / \mathrm{s}$ ) in which the surface pressure for different interfacial areas of the pendant drop were obtained. Finally, after the growing and shrinking cycles, the interfacial dilatational rheology was measured by performing periodic injections and extractions of $1 \mu \mathrm{L}$ of the silicone drop phase at different periods (1 s, $5 \mathrm{~s}$ and $10 \mathrm{~s}$ ), thus obtaining the interfacial dilatational elastic modulus, $E_{d}$ and viscosity modulus, $\eta_{\mathrm{d}}$.

For hydrophobic fumed silica particles (14\% SiOH), the compression and expansion cycles are plotted in Figure 15(a) for different particle concentrations before the exchange of the silicone oil drop phase. As can be seen, the surface pressure $\left(\Pi=\gamma_{0}-\gamma\right.$, where $\gamma_{0}=1.46 \mathrm{mN} / \mathrm{m}$ is the interfacial tension of the bare sunflower oil-20 cS PDMS silicone oil interface measured with the pendant drop) increases 
with particle concentration and with the compression of the interface. The high hysteresis and open cycles (no overlap of either compression or expansion cycles) of the two highest concentrations suggest that the particles were leaving the interface upon compression. This was proven with the pendant drop exchange which produced closed (compression and expansion cycles overlap) hysteresis cycles as can be seen in Figure 15(b). The results suggest that once drop exchange occurs, the particles are irreversibly attached to the interface, since the drop exchange with pure silicone oil doesn't produce a lower interfacial activity. The highest concentration here of $0.47 \mathrm{wt}$ \% in the silicone oil is comparable to $1 \mathrm{wt} . \%$ in a 1:1 mixture of silicone oil and vegetable oil reported in ref. [9]. Finally, we performed interfacial dilatational rheology by sinusoidal injections and extractions of $1 \mu \mathrm{L}$ at different periods. This was also performed at different pendant drop volumes to obtain different particle layer compression states (see Figure 16). It is worth mentioning that although the rheological measurements were performed after exchange of the drop phase, we still refer to the concentrations in the drop phase before exchange. Taking the example of the $5 \mu \mathrm{L}$ pendant drop, it can be seen that the elasticity (see Figure 16(a)) decreases for increasing periods. The opposite can be observed with the viscosity, which is very low compared to the elasticity. Moreover, although the elasticity and viscosity are approximately the same for very low initial particle concentration, the differences become more visible as the interface becomes more compressed. At $5 \mu \mathrm{L}$, there is an increase of one order of magnitude in the elasticity and the viscosity, pointing out that this particle layer is highly elastic.

We also characterised the PF-5 Eight Pearl 300S-Al platelet particles at the sunflower oil-20 cS PDMS silicone oil interface. The first finding was that the interfacial tension was still changing after 5 min compared to that with silica particles (see Figure 17(a)). Thus, when the pendant drop was kept at a constant volume of $40 \mu \mathrm{L}$ during 5 min the interfacial pressure increased significantly. Notice in Figure 17(a) that the interfacial activity was higher after exchange, probably because the time elapsed during the exchange enabled more particles to reach the interface. However, after $80 \mathrm{~min}$ (measurement 2), the interfacial pressure was similar before and after exchange. This points out that these larger microparticles need more time to reach and coat the interface, although once there they become irreversibly attached. Once more, the interfacial activity was high with interfacial pressures of $1.3 \mathrm{mN} / \mathrm{m}$, corresponding to an interfacial tension of $0.16 \mathrm{mN} / \mathrm{m}$ which is nine times lower than the bare interface. This interfacial activity again increased with initial particle concentration (see Figure 17(b)). The interfacial dilatational rheology was impossible to be measured for the $5 \mu$ pendant drop because of the buckling of the interface making it impossible to extract the profile of the pendant drop. Moreover, the elastic and viscosity moduli for the $20 \mu \mathrm{L}$ and $40 \mu \mathrm{L}$ pendant drops (not shown) presented similar behaviour as the silica particles with $E_{d}<8 \mathrm{mN} / \mathrm{m}$ and $\eta_{\mathrm{d}}<0.03 \mathrm{mN} / \mathrm{m} \mathrm{s}$ again demonstrating elastic behaviour. Nevertheless, unlike silica particles which displayed a clear interface before and after the growing and shrinking cycles (see Figure 18(a)), the PF-5 platelet particles 
exhibited a change in the appearance of the interface when the growing and shrinking cycles were performed, where a breakage of the interfacial layer is observed from Figure 18(b) to 18(c). Thus, our results reinforce the idea of the hydrophobic particles being excellent in the stabilization of oil-oil emulsions as they exhibit irreversible adsorption, high interfacial activity and elastic shell behaviour.

\section{Conclusions}

The fundamental physics of particles adsorbed at liquid interfaces is a wide field of study due to its numerous applications and in particular in emulsion science. Recently, there has been a substantial improvement in the understanding of the microstructure of particles at liquid interfaces, for example characterising the contact angle of each single particle attached to a given interface. Although this is the case for water-air surfaces and water-oil interfaces, the case of non-aqueous liquid-liquid interfaces still remains poorly understood. Non-aqueous emulsions in which the water phase is replaced with another polar liquid (i.e. one with a relatively high dielectric constant) retain similar behaviour as the traditional water-oil emulsions. Non-aqueous immiscible polymer blends are of great interest in industry and the addition of inorganic particles enables one to choose the interfacial characteristics of the blend, despite the need for higher temperatures required to melt the polymers. Oil-oil interfaces, where both oils are immiscible and of low dielectric constant (typically $\varepsilon<3$ ), are scarcely studied in the literature and here we compile the main recent work devoted to such interfaces. In particular, the sunflower oil-silicone oil interface is studied in the presence of hydrophobic silica or clay particles, including an original pendant drop tensiometry study. The main conclusions regarding particles adsorbed at oil-oil interfaces are that the best particles to stabilise oiloil emulsions are highly hydrophobic, exhibiting irreversible adsorption, high interfacial activity and elastic shell behaviour.

\section{Acknowledgements}

This work was supported by the Spanish MINECO (projects MAT2013-44429-R and MAT201460615R), by "Junta de Andalucía" and FEDER (projects P10-FQM-5977 and P12-FQM-1443). The authors thank Dr. J.A. Holgado-Terriza and Dr. J.L. Muros-Cobos for the software Contacto ${ }^{\circledR}$ used for surface tension measurements. 


\section{References}

1. Nushtaeva AV. Superstabilization of emulsions by solid particles. Colloids Surf. A 2015; 481:283-7.

2. Whitby CP, Wanless EJ. Controlling Pickering Emulsion Destabilisation: A Route to Fabricating New Materials by Phase Inversion. Materials 2016;9: doi.org/10.3390/ma9080626.

3. Lee LL, Niknafs N, Hancocks RD, Norton IT. Emulsification: Mechanistic understanding. Trends Food Sci. Tech. 2013; 31:72-8.

4. Kronberg B, Holmberg K, Lindman B. Emulsions and Emulsifiers. In Surface Chemistry of Surfactants and Polymers; John Wiley \& Sons, Chichester, 2014, pp. 431-45.

5. Xiao J, Li Y, Huang Q. Recent advances on food-grade particles stabilized Pickering emulsions: Fabrication, characterization and research trends. Trends Food Sci. Tech. 2016; 55:48-60.

6. Duffus LJ, Norton JE, Smith P, Norton IT, Spyropoulos F. A comparative study on the capacity of a range of food-grade particles to form stable $\mathrm{O} / \mathrm{W}$ and W/O Pickering emulsions. J. Colloid Interface Sci. 2016; 473:9-21.

7. Tsabet E, Fradette L. Effect of the properties of oil, particles, and water on the production of Pickering emulsions. Chem. Eng. Res. Des. 2015; 97:9-17.

8. Crespy D, Landfester K. Making dry fertile: a practical tour of non-aqueous emulsions and miniemulsions, their preparation and some applications. Soft Matter 2011;7:11054-64.

9. Binks BP, Tyowua AT. Oil-in-oil emulsions stabilised solely by solid particles. Soft Matter 2016; 12:876-87.

10. Petersen RV, Hamill RD. Studies on Nonaqueous Emulsions. J. Soc. Cosmet. Chem. 1968;19:627-40.

11. Binks BP, Fletcher PDI, Thompson MA. Influence of Propylene Glycol on Aqueous Silica Dispersions and Particle-Stabilized Emulsions. Langmuir 2013; 29:5723-33.

12. Dyab AKF, Al-Haque HN. Particle-stabilised non-aqueous systems. RSC Adv. 2013; 3:13101-5.

13. Tawfeek AM, Dyab AKF, Al-Lohedan HA. Synergetic Effect of Reactive Surfactants and Clay Particles on Stabilization of Nonaqueous Oil-in-Oil (o/o) Emulsions. J. Disper. Sci. Technol. 2014; 35:265-72.

14. Dyab AKF, Atta AM. Microgel-stabilised non-aqueous emulsions. RSC Adv. 2013; 3:25662-5.

15. Datta SS, Gerrard DD, Rhodes TS, Mason TG, Weitz DA. Rheology of attractive emulsions. Phys. Rev. E 2011; 84:041404.

16. Imhof A, Pine DJ. Uniform Macroporous Ceramics and Plastics by Emulsion Templating. Adv. Mater. 1998;10(9):697-700.

17. Sakthivel T, Jaitely V, Patel NV, Florence AT. Non-aqueous emulsions: hydrocarbon-formamide systems. Int. J. Pharm. 2001;214(1-2):43-8.

18. Rizzelli SL, Jones ER, Thompson KL, Armes SP. Preparation of non-aqueous Pickering emulsions using anisotropic block copolymer nanoparticles. Colloid Polym. Sci. 2016; 294:1-12.

19. Atanase LI, Riess G. Stabilization of non-aqueous emulsions by poly(2-vinylpyridine)-bpoly(butadiene) block copolymers. Colloids Surf. A 2014; 458:19-24.

20. Atanase LI, Riess G. PEG 400/Paraffin Oil Non-Aqueous Emulsions Stabilized by PBut-Block-P2VP Block Copolymers. J. Appl. Polym. Sci. 2015; 132: doi.org/10.1002/app.41390.

21. Atanase LI, Riess G. Block copolymer stabilized nonaqueous biocompatible sub-micron emulsions for topical applications. Int. J. Pharm. 2013; 448: 339-45.

22. Voigt M, Koerber M, Bodmeier R. Improved physical stability and injectability of non-aqueous in situ PLGA microparticle forming emulsions. Int. J. Pharm. 2012; 434:251-6.

23. Periard J, Banderet A, Riess G. Emulsifying effect of block and graft copolymers-oil in oil emulsions. J. Polym. Sci. B Polym. Lett. 1970;8:109-114.

24. Atanase LI, Riess G. Block copolymers as polymeric stabilizers in non-aqueous emulsion polymerization. Polym. Int. 2011;60:1563-1573. 
25. Bai L, Fruehwirth JW, Cheng X, Macosko CW. Dynamics and rheology of nonpolar bijels. Soft Matter 2015; 11:5282-93.

26. Causa A, Filippone G, Domingo C, Salerno A. Study of the morphology and texture of poly(3caprolactone)/polyethylene oxide blend films as a function of composition and the addition of nanofillers with different functionalities. RSC Adv. 2015; 5:59354-63.

27. Laoutid F, Picard A, Persenaire O, Dubois P. Investigation of the alumina nanoparticle role in the enhancement of the mechanical properties of polyamide/polycarbonate blends. Polym. Degrad. Stab. 2015; 112:137-44.

28. Liu X, Dong X, Liu W, Xing Q, Zou F, Han CC, Wang D, Liang A, Li C, Xie X. Characterization on the phase separation behaviour of styrene-butadiene rubber/polyisoprene/organoclay ternary blends under oscillatory shear. J. Chem. Phys. 2015; 143; 114903-1 to 11.

29. Maani A, Carreau PJ. Rheological and morphological properties of thermoplastic olefin blends containing nanosilica. J. NonNewton. Fluid Mech. 2016; 233:95-106.

30. Mao C, Kong M, Yang Q, Li G, Huang Y. Droplet coalescence and clustering behaviour in microsphere-filled polymeric emulsions under shear flow: the key role of asymmetric interfacial affinities. Phys. Chem. Chem. Phys. 2016; 18:4429-36.

31. Mao C, Kong M, Yang Q, Li G, Huang Y. Vorticity Deformation in Polymeric Emulsions Induced by Anisotropic Ellipsoids. ACS Macro Lett. 2016; 5:900-3.

32. Moghimi E, Goharpey F, Foudazi R. Role of droplet bridging on the stability of particle-containing immiscible polymer blends. Rheol. Acta 2014; 53:165-80.

33. Pawar S.P., Bose S. Peculiar morphological transitions induced by nanoparticles in polymeric blends: retarded relaxation or altered interfacial tension? Phys. Chem. Chem. Phys. 2015; 17:14470-8.

34. Qian J, Xiao Z, Dong L, Tang D, Li M, Yang Q, Huang Y, Liao X. Morphology and crystallization behaviour of PCL/SAN blends containing nanosilica with different surface properties. J. Appl. Polym. Sci. 2016; 133: doi.org/10.1002/app.44157.

35. Sangroniz L, Palacios JK, Fernández M, Eguiazabal JI, Santamaria A, Müller AJ. Linear and nonlinear rheological behaviour of polypropylene/polyamide blends modified with a compatibilizer agent and nanosilica and its relationship with the morphology. Eur. Polym. J. 2016; 83:10-21.

36. Salehiyan R, Song HY, Kim M, Choi WJ, and Hyun K. Morphological Evaluation of PP/PS Blends Filled with Different Types of Clays by Nonlinear Rheological Analysis. Macromol. 2016; 49:314860.

37. Salehiyan R, Song HY, Choi WJ, Hyun K. Characterization of Effects of Silica Nanoparticles on (80/20) PP/PS Blends via Nonlinear Rheological Properties from Fourier Transform Rheology. Macromol. 2015; 48:4669-79.

38. Trifkovic M, Hedegaard AT, Sheikhzadeh M, Huang S, Macosko CW. Stabilization of PE/PEO Cocontinuous Blends by Interfacial Nanoclays. Macromol. 2015; 48:4631-44.

39. Zou ZM, Sun ZY, An LJ. Effect of fumed silica nanoparticles on the morphology and rheology of immiscible polymer blends. Rheol. Acta 2014; 53:43-53.

40. Fenouillot F, Cassagnau P, Majesté JC. Uneven distribution of nanoparticles in immiscible fluids: Morphology development in polymer blends. Polymer 2009; 50:1333-50.

41. Taguet A, Cassagnau P, Lopez-Cuesta JM. Structuration, selective dispersion and compatibilizing effect of (nano)fillers in polymer blends. Prog. Polym. Sci. 2014; 39:1526-63.

42. Salzano de Luna M, Filippone G. Effects of nanoparticles on the morphology of immiscible polymer blends. Eur. Polym. J. 2016; 79:198-218.

43. Atanase LI, Riess G. Water-dispersible non-aqueous emulsions stabilized by a poly(butadiene)-bpoly(2-vinylpyridine) block copolymer. C. R. Chim. 2014;17:310-5.

44. Atanase LI, Lerch JP, Riess G. Water dispersibility of non-aqueous emulsions stabilized and viscosified by a poly(butadiene)-poly(2-vinylpyridine)-poly(ethylene oxide) (PBut-P2VP-PE0) 
triblock copolymer. Colloids Surf. A 2015;464:89-95.

45. Binks BP. Particles as surfactants-similarities and differences. Curr. Opin. Colloid Interface Sci. 2002; 7:21-41.

46. Geisel K, Isa L, Richtering W. Unraveling the 3D Localization and Deformation of Responsive Microgels at Oil/Water Interfaces: A Step Forward in Understanding Soft Emulsion Stabilizers. Langmuir 2012; 28:15770-6.

47. Maestro A, Guzmán E, Ortega F, Rubio RG. Contact angle of micro- and nanoparticles at fluid interfaces. Curr. Opin. Colloid Interface Sci. 2014; 19:355-67.

48. Neumann AW, David R, Zuo Y. Applied surface thermodynamics. CRC Press, Taylor \& Francis, Boca Raton, 1996.

49. McBride SP, Law BM. Influence of line tension on spherical colloidal particles at liquid-vapor interfaces. Phys. Rev. Lett. 2012; 109: 196101-1 to 5.

50. Fernandez-Rodriguez MA, Rodriguez-Valverde MA, Cabrerizo-Vilchez MA, Hidalgo-Alvarez R. Surface activity of Janus particles adsorbed at fluid-fluid interfaces: Theoretical and experimental aspects. Adv. Colloid Interface Sci. 2016; 233:240-54.

51. Zeng M, Mi J, Zhong C. Wetting behaviour of spherical nanoparticles at a vapor-liquid interface: a density functional theory study. Phys. Chem. Chem. Phys. 2011; 13:3932-41.

52. Garbin V, Crocker JC, Stebe KJ. Nanoparticles at fluid interfaces: Exploiting capping ligands to control adsorption, stability and dynamics. J. Colloid Interface Sci. 2012; 387:1-11.

53. Kumar A, Park BJ, Tu F, Lee D. Amphiphilic Janus particles at fluid interfaces. Soft Matter 2013; 9:6604-17.

54. Fernandez-Rodriguez MA, Chen L, Deming CP, Rodriguez-Valverde MA, Chen S, Cabrerizo-Vilchez MA, Hidalgo-Alvarez R. A simple strategy to improve the interfacial activity of true Janus gold nanoparticles: a shorter hydrophilic capping ligand. Soft Matter 2016; 12:31-4.

55. Binks BP, Fletcher PDI. Particles Adsorbed at the Oil-Water Interface:A Theoretical Comparison between Spheres of Uniform Wettability and Janus Particles. Langmuir 2001; 17:4708-10.

56. Stamou D, Duschl C, Johannsmann D. Long-range attraction between colloidal spheres at the airwater interface: The consequence of an irregular meniscus. Phys. Rev. E 2000; 62:5263-72.

57. Nikolaides MG, Bausch AR, Hsu MF, Dinsmore AD, Brenner MP, Gay C, Weitz DA. Electric-fieldinduced capillary attraction between like-charged particles at liquid interfaces. Nature 2002; 420:299-301.

58. Danova KD, Kralchevsky PA, Naydenova BN, Brennb G. Interactions between particles with an undulated contact line at a fluid interface: Capillary multipoles of arbitrary order. J. Colloid Interface Sci. 2005; 287:121-34.

59. Holgado-Terriza JA, Gómez-Lopera JF, Luque-Escamilla PL, Atae-Allah C, Cabrerizo-Vílchez MA. Measurement of ultralow interfacial tension with ADSA using an entropic edge-detector. Colloids Surf. A 1999; 156:579-586.

60. Paunov VN. Novel method for determining the three-phase contact angle of colloid particles adsorbed at air-water and oil-water interfaces. Langmuir 2003; 19:7970-6.

61. Isa L, Lucas F, Wepf R, Reimhult E. Measuring single-nanoparticle wetting properties by freezefracture shadow-casting cryo-scanning electron microscopy. Nat. Commun. 2011; 2:438, 1-9.

62. Fernandez-Rodriguez MA, Ramos J, Isa L, Rodriguez-Valverde MA, Cabrerizo-Vilchez MA, HidalgoAlvarez R. Interfacial activity and contact angle of homogeneous, functionalized, and Janus nanoparticles at the water/decane interface. Langmuir 2015; 31:8818-8823.

63. Anachkov SE, and Lesov I, Zanini M, Kralchevsky PA, Denkov ND, Isa L. Particle detachment from fluid interfaces: Theory vs. Experiments. Soft Matter 2016; DOI:10.1039/C6SM01716A.

64. Reguera, J, Ponomarev E, Geue T, Stellacci F, Bresme F, Moglianetti M. Contact angle and adsorption energies of nanoparticles at the air-liquid interface determined by neutron reflectivity and molecular dynamics. Nanoscale 2015; 7:5665-73. 
65. Clark JN, Beitra L, Xiong G, Higginbotham A, Fritz DM, Lemke HT, Zhu D, Chollet M, Williams GJ, Messerschmidt M, Abbey B, Harder RJ, Korsunsky AM, Wark JS, Robinson IK. Ultrafast ThreeDimensional Imaging of Lattice Dynamics in Individual Gold Nanocrystal. Science 2013; 341:56-9.

66. Latychevskaia T, Mancini GF, Carbone F. The role of the coherence in the cross-correlation analysis of diffraction patterns from two-dimensional dense mono-disperse systems. Sci. Rep. 2015; 5; doi.org/10.1038/srep16573.

67. Mancini GF, Latychevskaia T, Pennacchio F, Reguera J, Stellacci F, Carbone F. Order/Disorder Dynamics in a Dodecanethiol-Capped Gold Nanoparticles Supracrystal by Small-Angle Ultrafast Electron Diffraction. Nano Lett. 2016; 16:2705-13.

68. Fameau AL, Lam S, Arnould A, Gaillard C, Velev OD, Saint-Jalmes A. Smart Nonaqueous Foams from Lipid-Based Oleogel. Langmuir 2015; 31:13501-10.

69. Binks BP, Garvey EJ, Vieira J. Whipped oil stabilised by surfactant crystals. Chem. Sci. 2016; 7:2621-32.

70. Crespy D, Landfester K. Making dry fertile: a practical tour of non-aqueous emulsions and miniemulsions, their preparation and some applications. Soft Matter 2011; 7:11054-64.

71. Marcel Rey M, Fernandez-Rodriguez MA, Steinacher M, Scheidegger L, Geisel K, Richtering W, Squires TM, Isa L. Isostructural solid-solid phase transition in monolayers of soft core-shell particles at fluid interfaces: structure and mechanics. Soft Matter 2016; 12:3545-57.

72. Pinaud F, Geisel K, Mass P, Catargi B, Isa L, Richtering W, Ravaine V, Schmitt V. Adsorption of microgels at an oil-water interface: correlation between packing and 2D elasticity. Soft Matter 2014; 10:6963-74.

73. Bonham JA, Faers MA, Duijneveldt JS. Non-aqueous microgel particles: synthesis, properties and applications. Soft Matter 2014; 10:9384-98.

74. Hu C, Wang Q, Ma C, Xia Q. Non-aqueous self-double-emulsifying drug delivery system: A new approach to enhance resveratrol solubility for effective transdermal delivery. Colloids Surf. A 2016; 489:360-9.

75. Lin Z, Schulz Jr WJ, Smith JR. Oil-in-oil and three-phase emulsions. US Pat. 6238657 B1, Dow Corning Corporation, 2001.

76. Nonomura Y, Sugawara T, Kashimoto A, Fukuda K, Hotta H Tsujii K. Self-Assembly of SurfaceActive Powder at the Interfaces of Selective Liquids. 1: Multiple Structural Polymorphism. Langmuir 2002; 18:10163-7.

77. Nonomura Y, Fukuda K, Komura S, Tsujii K. Self-Assembly of Surface-Active Powder at the Interfaces of Selective Liquids. 2: Behaviour of an Organic-Crystalline Powder. Langmuir 2003; 19:10152-6.

78. Vartanian RD. Stable mineral oil-silicone oil compositions. US Pat. 3445385, American Cyanamid Company, 1969.

79. Greene GH, Phillips JC, Stults JF. Non-aqueous emulsion of silicone oil and stearine, W01991010771 A1, Karlshamns AB, 1991.

80. Lannibois-Drean H, Ricca JM, Destarac M, Olier P. Oil-in-oil emulsions comprising a silicone, dispersions and use of said emulsions, W003/000396 A1, Rhodia Chimie, 2003.

81. Pan XD, McKinley GH. Characteristics of Electrorheological Responses in an Emulsion System. J. Colloid Interface Sci. 1997; 195:101-13.

82. Loudet JC, Richard H, Sigaud G, Poulin P. Nonaqueous Liquid Crystal Emulsions. Langmuir 2000; 16:6724-30.

83. Jones TK, Nair M. Oil-in-oil dispersions stabilised by solid particles and methods of making the same, US Pat. 8323392 B2, Eastman Kodak Company, 2012.

84. Jaitely V, Sakthivel T, Magee G, Florence AT. Formulation of oil in oil emulsions: potential drug reservoirs for slow release. J. Drug Delivery Sci. Technol. 2004; 14:113-7.

85. Verma S, Dangi JS. Non aqueous microemulsions: Ideal vehicles for lipophilic drugs. Ind. J. Novel 
Drug Del. 2012; 4:223-6.

86. Suitthimeathegorn 0 , Jaitely V, Florence AT. Novel anhydrous emulsions: formulation as controlled release vehicles. Int. J. Pharm. 2005;298(2):367-71.

87. Guillaume A, Sagi F. Homogeneous dispersions of diorganopolysiloxane compositions in mineral oils, US Pat. 4115343, Rhone-Poulenc Industries, 1978.

88. Cauvin S, Colson A, Dimitrova T, Lecomte JP. Silicone Dispersions. W02010072711 A1, Dow Corning Corporation, 2010.

89. Nair M, Jones TK, Brick MC. Oil-in-oil emulsions, US Pat. 2007/0189998 A1, Eastman Kodak Company, 2007.

90. Holtze C, Guerra RE, Agresti J, Weitz DA, Ahn K, Hutchison JB, Griffiths A, Harrak AE, Miller OJ, Baret JC, Taly V, Ryckelynck M, Merten C. Fluorocarbon emulsion stabilizing surfactants, W02008/021123 A1, Harvard College, 2008. 
Table 1. Summary of the published literature on emulsions of two immiscible oils both of dielectric constant $<3.5$. Taken from ref. 7 .

\begin{tabular}{|c|c|c|c|c|}
\hline Dispersed phase & Continuous phase & Emulsifier & Application & Ref. \\
\hline $\begin{array}{c}\text { Silicone oil (PDMS, }{ }^{\text {a }} \\
\text { PMPS }^{b} \text { ) }\end{array}$ & Mineral oil & $\begin{array}{c}\text { Hydrophobic } \\
\text { bentonite particles }\end{array}$ & $\begin{array}{l}\text { Lubricants on } \\
\text { fibers }\end{array}$ & {$[78]$} \\
\hline $\begin{array}{l}\text { Silicone oil, >300 cS } \\
\text { (polydialkylsiloxane) }\end{array}$ & Mineral oil & $\begin{array}{c}\text { Ethylene-vinyl } \\
\text { acetate copolymer }\end{array}$ & $\begin{array}{l}\text { Foam inhibitor for } \\
\text { lubricating oils }\end{array}$ & {$[87]$} \\
\hline $\begin{array}{l}\text { Triglyceride oil } \\
\text { (soybean, } \\
\text { rapeseed...) }\end{array}$ & $\begin{array}{l}\text { Silicone oil, }<500 \\
\text { cS (PDMS) }\end{array}$ & Silicone surfactant & Lubricant on fibers & {$[79]$} \\
\hline $\begin{array}{c}\text { Silicone oil, } 2500 \mathrm{cS} \\
\text { (PDMS) }\end{array}$ & $\begin{array}{c}\text { Chlorinated } \\
\text { paraffin oil, } 340 \mathrm{cS}\end{array}$ & $\begin{array}{c}\text { None (drops had } \\
\text { short lifetimes) }\end{array}$ & \multirow{2}{*}{$\begin{array}{l}\text { Electro-rheological } \\
\text { fluid }\end{array}$} & \multirow{2}{*}[81]{} \\
\hline $\begin{array}{c}\text { Chlorinated paraffin } \\
\text { oil, } 340 \mathrm{cS}\end{array}$ & $\begin{array}{l}\text { Silicone oil, } 2500 \\
\text { cS (PDMS) }\end{array}$ & $\begin{array}{l}\text { None (drops had } \\
\text { short lifetimes) }\end{array}$ & & \\
\hline $\begin{array}{c}\text { Silicone oil, } 1000 \mathrm{cS} \\
\text { (PDMS) }\end{array}$ & $\begin{array}{l}\text { Thermotropic } \\
\text { liquid crystal }^{\mathrm{c}}\end{array}$ & $\begin{array}{l}\text { Cyanobiphenyl- } \\
\text { PDMS oligomer }\end{array}$ & \multirow{2}{*}{$\begin{array}{l}\text { Electro-optical } \\
\text { display devices }\end{array}$} & \multirow[b]{2}{*}[82]{} \\
\hline $\begin{array}{c}\text { Thermotropic liquid } \\
\text { crystal }\end{array}$ & $\begin{array}{l}\text { Silicone oil, } 1000 \\
\text { cS (PDMS) }\end{array}$ & $\begin{array}{l}\text { Cyanobiphenyl- } \\
\text { PDMS polymer }\end{array}$ & & \\
\hline $\begin{array}{l}\text { Mineral oil or } \\
\text { vegetable oil }\end{array}$ & $\begin{array}{c}\text { Silicone oil, }<1000 \\
\text { cS }\end{array}$ & $\begin{array}{c}\text { Elastomeric } \\
\text { silicone polyether }\end{array}$ & $\begin{array}{l}\text { Personal care and } \\
\text { cosmetics }\end{array}$ & {$[75]$} \\
\hline Silicone oil (PDMS) & PFPMIE $^{d}$ & $\begin{array}{c}\text { Fluorinated } \\
\text { silicone resin } \\
\text { particles (sphere) }\end{array}$ & \multirow{2}{*}{ Cosmetics } & {$[76]$} \\
\hline Silicone oil (PDMS) & PFPMIE & $\begin{array}{l}\text { Fluorinated } \mathrm{Ca} \\
\text { lauroyl taurate } \\
\text { particles (plate) }\end{array}$ & & {$[77]$} \\
\hline $\begin{array}{l}\text { Mineral oil or animal } \\
\text { or vegetable oil }\end{array}$ & $\begin{array}{l}\text { Silicone oil } \\
\text { (PDMS) }\end{array}$ & $\begin{array}{c}\text { Block copolymer, } \\
\left.\text { e.g. p(BA }{ }^{\mathrm{e}}\right)- \\
\text { PDMS-p(BA) }\end{array}$ & Personal care & {$[80]$} \\
\hline Castor oil & $\begin{array}{l}\text { Silicone oil, }<100 \\
\text { cS (PDMS) }\end{array}$ & $\begin{array}{l}\text { Nonionic } \\
\text { surfactant } \\
\left(\mathrm{O} / \mathrm{NPE}^{\mathrm{f}}\right)\end{array}$ & $\begin{array}{l}\text { Pharmaceutical } \\
\text { formulations }\end{array}$ & {$[84]$} \\
\hline Organic phosphate & Hydrocarbon & $\begin{array}{c}\text { Diblock or triblock } \\
\text { copolymer }\end{array}$ & $\begin{array}{c}\text { Liquid toning } \\
\text { systems }\end{array}$ & {$[89]$} \\
\hline Hydrocarbon & Fluorocarbon & $\begin{array}{c}\text { Fluorocarbon } \\
\text { surfactant }\end{array}$ & $\begin{array}{c}\text { Vehicles for } \\
\text { chemical reactions }\end{array}$ & {$[90]$} \\
\hline (Silica in) PDMS & $\begin{array}{l}\text { Vegetable oil or } \\
\text { PEO-PPO } \\
\text { copolymer }\end{array}$ & Wax crystals & Antifoam & {$[88]$} \\
\hline $\begin{array}{l}\text { Organic phosphate } \\
\text { or silicone oil }\end{array}$ & $\begin{array}{l}\text { Paraffin oil or } \\
\text { white mineral oil } \\
\text { or cyclic silicone }\end{array}$ & $\begin{array}{l}\text { Hydrophobic } \\
\text { fumed silica } \\
\text { particles + polymer } \\
\text { co-stabiliser }\end{array}$ & $\begin{array}{c}\text { Electro-optical } \\
\text { modulating display } \\
\text { devices }\end{array}$ & {$[83]$} \\
\hline Castor oil & Silicone oil, $20 \mathrm{cS}$ & \multirow{2}{*}{ Silicone surfactant } & \multirow[b]{2}{*}{$\begin{array}{l}\text { Pharmaceutical } \\
\text { formulations }\end{array}$} & \multirow[b]{2}{*}[85]{} \\
\hline $\begin{array}{l}\text { Silicone oil, } 20 \mathrm{cS} \\
\text { (PDMS) }\end{array}$ & Castor oil & & & \\
\hline
\end{tabular}

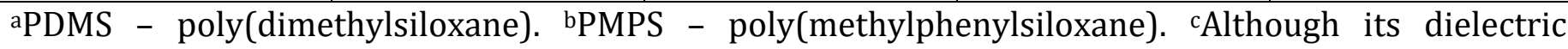
constant is $>3$ it is included as an interesting oil. dPFPMIE - perfluoropolymethylisopropyl ether. ${ }^{\text {eBA }}$ - butyl acrylate. ${ }^{\mathrm{O}} / \mathrm{NPE}$ - octyl/nonylpolyoxyethylene ether. 


\section{Figure legends}

Figure 1. (a) Sketch of a particle of radius $R$ adsorbed at a fluid-fluid interface exhibiting a contact angle $\theta$ (through fluid 1). $\gamma_{P f 1}$ is the interfacial tension particle-fluid $1, \gamma_{P f 2}$ is the interfacial tension particle-fluid 2, $\gamma_{f 1 f 2}$ is the fluid 1-fluid 2 interfacial tension and $\tau$ is the line tension. (b) Sketch of the position of a particle of increasing hydrophobicity. (c) Detachment energy of a spherical homogeneous particle from the interface, $\Delta \mathrm{E}_{\mathrm{p}}$ as a function of $\theta$ for particles of $\mathrm{R}=10 \mathrm{~nm}$ (left) and $\mathrm{R}=1 \mu \mathrm{m}$ (right) at $25^{\circ} \mathrm{C}\left(\gamma_{\mathrm{f} 1 \mathrm{f} 2}=50 \mathrm{mN} / \mathrm{m}\right)$. Reprinted from [47], copyright 2013 with permission from Elsevier.

Figure 2. Geometry of a Janus particle within an oil-water interface. The relative areas of the polar and apolar particle surface regions are parametrised by the angle $\alpha$. The immersion depth of the particle in the oil-water interface is parametrised by the angle $\beta$. Reprinted from [55], copyright 2001 with permission from American Chemical Society.

Figure 3. Variation of particle desorption energy with area-weighted average contact angle for particles of $R=10 \mathrm{~nm}$ and $\alpha=90^{\circ}$. The oil-water tension was set to $36 \mathrm{mN} \mathrm{m}^{-1}$. In order of increasing desorption energies, the curves refer to $\Delta \theta$ of 0 (the homogeneous particle case), 20, 40, 60 and $90^{\circ}$. Reprinted from [55], copyright 2001 with permission from American Chemical Society.

Figure 4. (a) Small pendant drop (volume $0.26 \mu \mathrm{L}$ ) of aqueous phase (more dense) in heptane (less dense) for the system 1-propanol-water-heptane. (b) Detection of the drop profile by the new entropic edge detector. Reprinted from [59], copyright 1999 with permission from Elsevier.

Figure 5. Low interfacial tension between water and heptane in the ternary system 1-propanolwater-heptane versus temperature. Reprinted from [59], copyright 1999 with permission from Elsevier.

Figure 6. FreSCa cryo-SEM images of (a) PMMA-HPs and (b) silica-FPs at the planar decane-water interface. The tungsten shadow projected by the nanoparticles enables estimating their contact angle. Adapted from [62], copyright 2015 with permission from American Chemical Society.

Figure 7. Pendant drops ( $5 \mu \mathrm{L})$ containing an aqueous suspension of $21.7 \times 10^{11}$ nanoparticles/mL of (a) PMMA-HPs and (b) silica-FPs both immersed in decane. The presence of fractal-like clusters of silica-FPs is clearly noticeable in the image. Reprinted from [62], copyright 2015 with permission from American Chemical Society. 
Figure 8. Scheme of the experimental setup for neutron reflectivity on a nanoparticle monolayer. (a) Nanoparticles form a monolayer at the air-water interface in a Langmuir trough. Neutron reflectivity is measured in situ on this monolayer using contrast variation of the aqueous subphase. (b) Model of the core-shell nanoparticles at the interface where $R$ and $l$ are the nanoparticle core radius and interparticle distance (measured by TEM), id is the immersion depth and $\rho(z)$ is the calculated scattering length density profile. Reproduced from [64], copyright 2015 with permission from The Royal Society of Chemistry.

Figure 9. Appearance of vessels after 6 months containing emulsions prepared from 50 vol.\% paraffin liquid and 50 vol.\% propylene glycol containing 1 wt.\% fumed silica particles of different \% SiOH content given . CPS = complete phase separation. Reprinted from [11], copyright 2013 with permission from American Chemical Society.

Figure 10. (a) Mean drop diameter vs particle hydrophobicity of emulsions formed in paraffin liquid (50 vol.\%) and propylene glycol (50 vol.\%) systems stabilised by $1 \mathrm{wt}$.\% fumed silica particles. (b) Optical microscopy images of emulsions in (a). Emulsions are glycol-in-oil for 14\% SiOH and oil-inglycol above this. Scale bars $=100 \mu \mathrm{m}$. Reprinted from [11], copyright 2013 with permission from American Chemical Society.

Figure 11. (a) Dynamic interfacial tension of formamide-paraffin oil interface in the presence of NIPAM/AMPS microgels at different concentrations given. (b) Evolution of pendant drop profile and interfacial coverage of a formamide drop in paraffin oil for different microgel concentrations in formamide at equilibrium. Reprinted from [14], copyright 2013 with permission from The Royal Society of Chemistry.

Figure 12. Cryo-SEM images of the polystyrene/polybutene bijel with hydrophobic silica nanoparticles (B-SNP) at the interface. (b) and (d) are magnified views of the rectangular regions indicated in (a) and (c). The white arrow indicates particles straddling or bridging two domains. The red arrows mark individual silica particles or a crater formed by one. The lines on the circled particle are through the cracks on each side of the particle, which likely run through the interface. The scale bar is $500 \mathrm{~nm}$. Reprinted from [25], copyright 2015 with permission from The Royal Society of Chemistry.

Figure 13. Photos after 1 month of glass vials containing (a and b) sunflower oil-20 cS PDMS (1:1) emulsions stabilised by 1 wt.\% of (a) DCDMS-coated and (b) fluoro-coated fumed silica particles and 
(c) olive oil-20 cS PDMS (1:1) emulsions stabilised by 1 wt.\% DCDMS-coated fumed silica particles. The fumed silica particles have different \% $\mathrm{SiOH}$ on their surfaces (given). Dashed lines indicate transitional phase inversion. Reprinted from [9], copyright 2016 with permission from the Royal Society of Chemistry.

Figure 14. (a) Photographs of glass vials containing sunflower oil-in-20 cS PDMS emulsions (1:1) one month after preparation stabilised by different concentrations (given, wt.\%) of PF-5 Eight Pearl 300SAl particles, (b) fraction of sunflower oil fsuno $(0)$ and fraction of PDMS oil f20cs PDMS $(\bigcirc)$ released from above emulsions versus particle concentration, (c) cryo-SEM image of a sunflower oil-in-50 cS PDMS emulsion stabilised by 1 wt.\% of silicone particles of PF-5 Tospearl 145A. Reprinted from [9], copyright 2016 with permission from the Royal Society of Chemistry.

Figure 15. (a) Interfacial pressure against area of the pendant drop at the sunflower oil-20 cS PDMS silicone oil interface for different concentrations of hydrophobic DCDMS-coated silica particles (14\% $\mathrm{SiOH}$ ) in the silicone oil drop. Each colour corresponds to a different experiment and the upper and lower curves correspond to the compressions and expansions of the interface, respectively. (b) Interfacial pressure against area of the pendant drop for the system in (a) showing the differences between the interface before and after exchange with pure 20 cS PDMS silicone oil. All the measurements were performed at room temperature of $25^{\circ} \mathrm{C}$.

Figure 16. (a) Interfacial dilatational elastic modulus $E_{d}$ and (b) interfacial viscosity modulus $\eta_{d}$ against the period of oscillation (for $1 \mu \mathrm{L}$ amplitude oscillation) for different concentrations of hydrophobic DCDMS-coated silica particles (14\% SiOH) at a sunflower oil-20 cS PDMS silicone oil interface and for different pendant drop volumes.

Figure 17. (a) Interfacial pressure against area of the pendant drop at the sunflower oil-20 cS PDMS silicone oil interface for a 0.47 wt.\% concentration of PF-5 Eight Pearl 300S-Al platelet particles in the silicone oil drop phase before and after exchange. The difference between the experiments corresponding to the red curves and the blue curves is the time that the pendant drop was kept constant at $40 \mu \mathrm{L}$ prior to the growing and shrinking cycles: $5 \mathrm{~min}$ for the red curves and $80 \mathrm{~min}$ for the blue curves. (b) Interfacial pressure against area of the pendant drop for the system in (a) for different particle concentrations after drop phase exchange and after $80 \mathrm{~min}$ of prior interfacial tension evolution. 
Figure 18. Photos of 20 cS PDMS silicone oil drops in sunflower oil containing 0.47 wt.\% of (a) hydrophobic DCDMS-coated fumed silica particles (14\% SiOH) and (b) PF-5 Eight Pearl 300S-Al platelet particles, after (a) 5 min and (b) 80 min of interfacial tension evolution and after exchange of the drop phase with pure silicone oil. (c) Same pendant drop as in (b) after the growing and shrinking cycles. 
Figure 1

a)

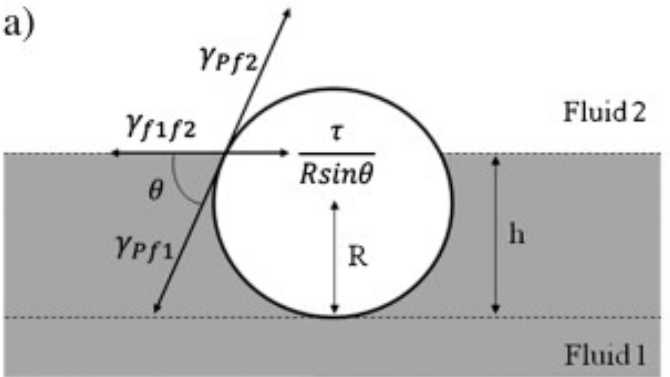

b)
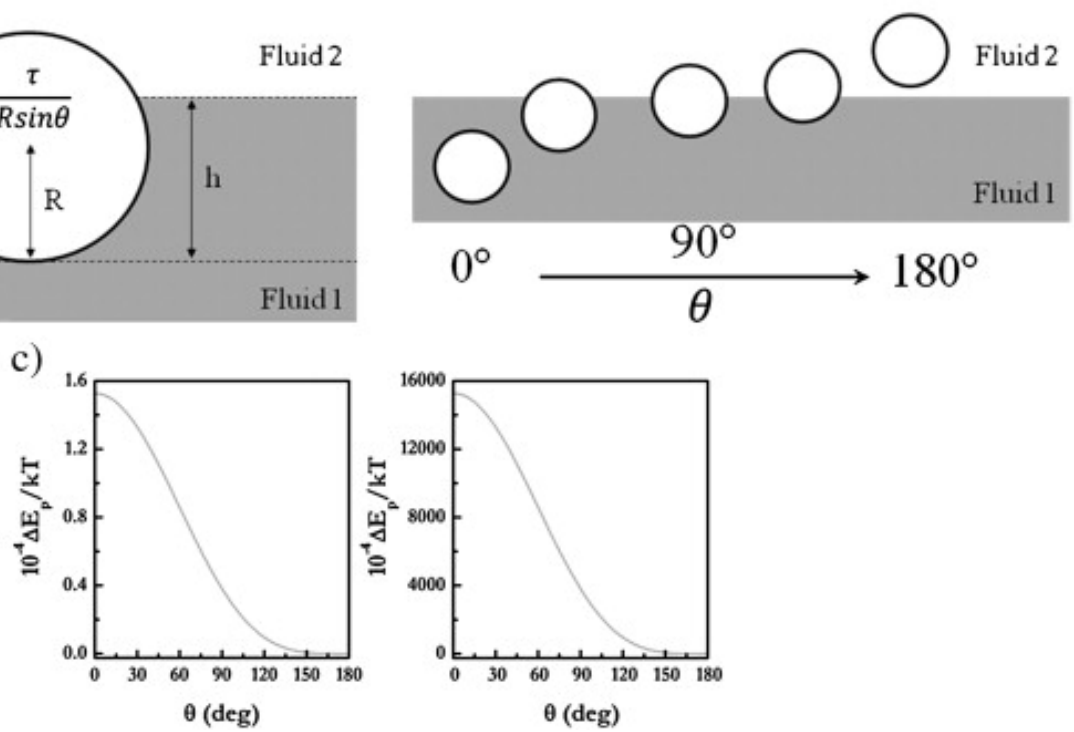

Figure 2

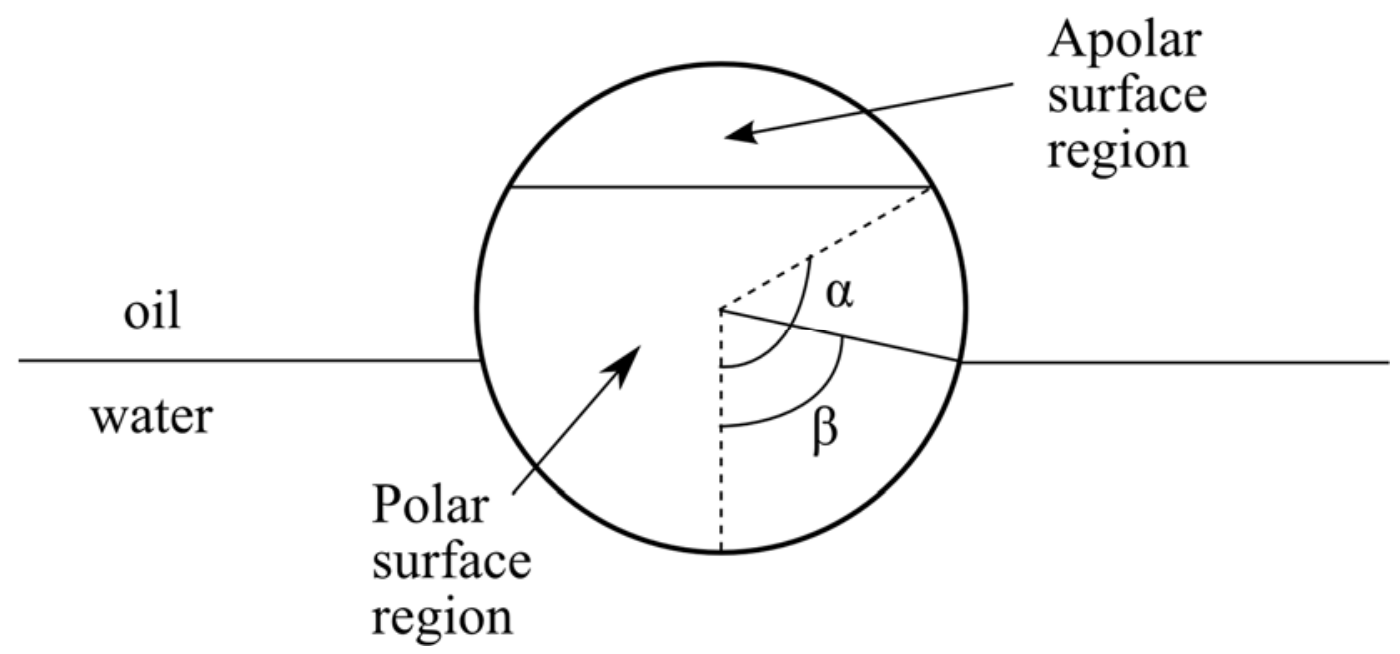


Figure 3

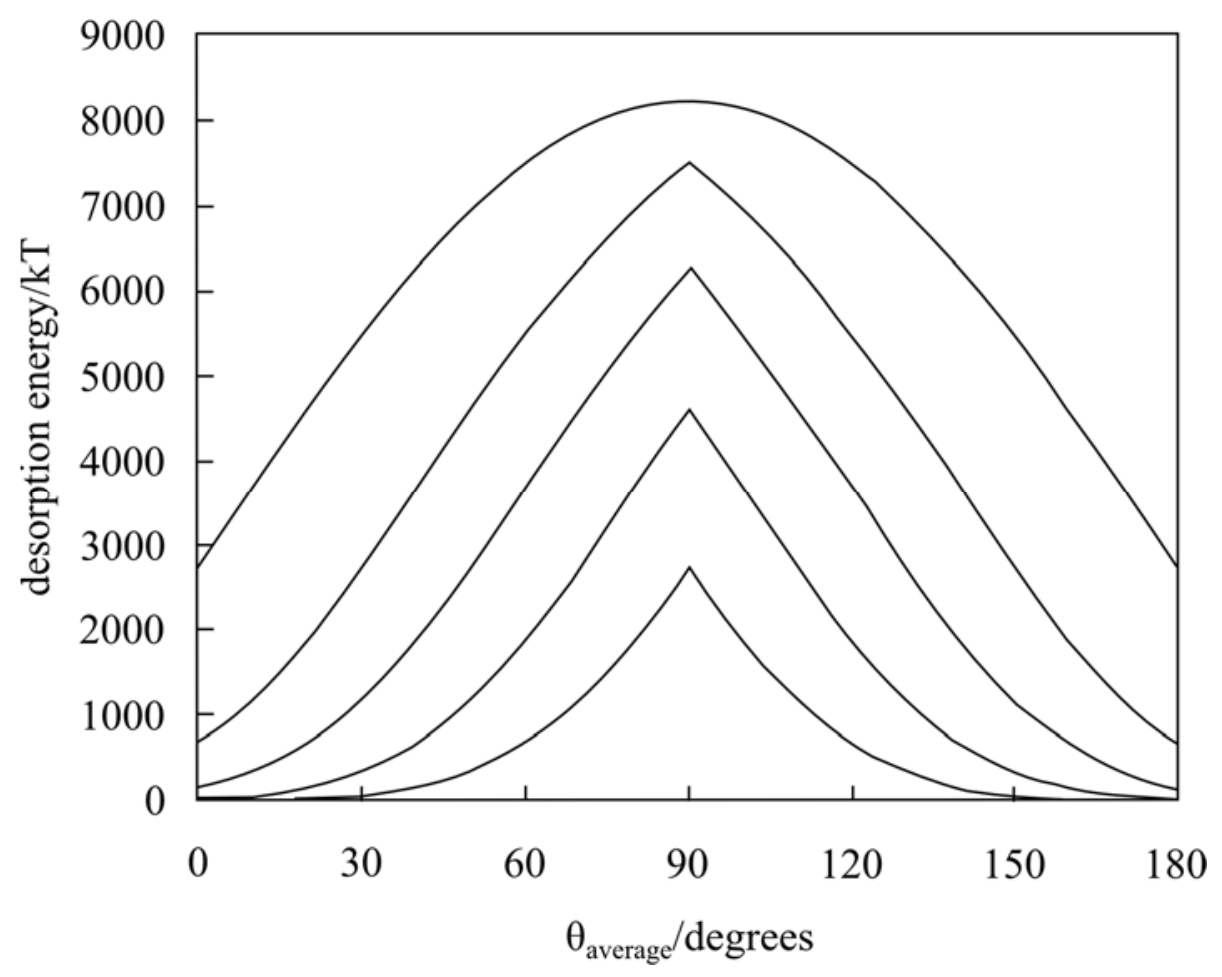

Figure 4

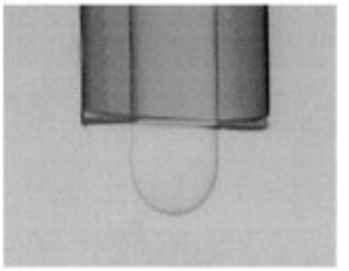

(a)

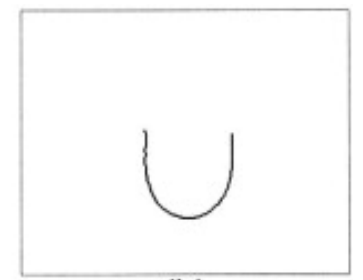

(b) 
Figure 5

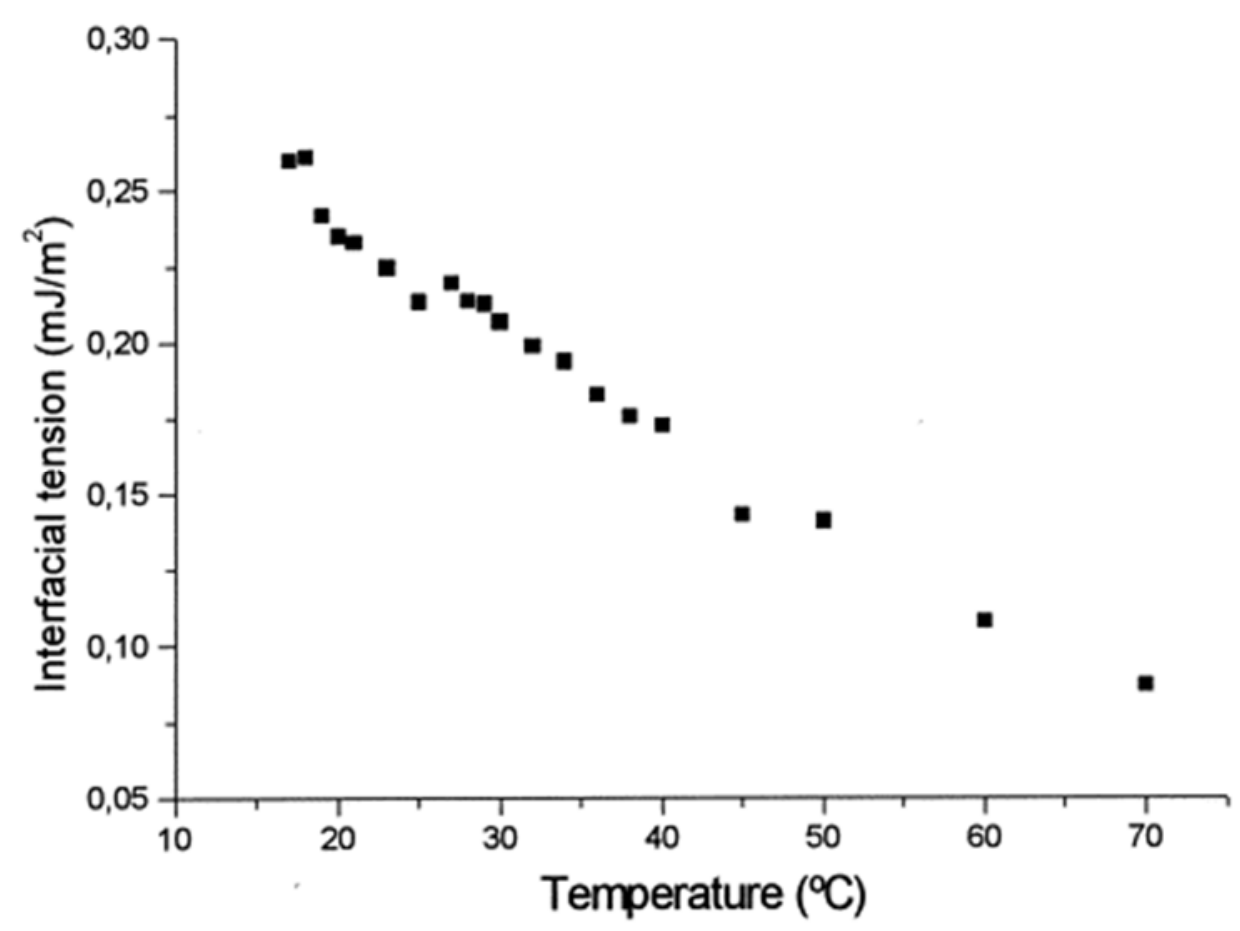

\section{Figure 6}

(a)

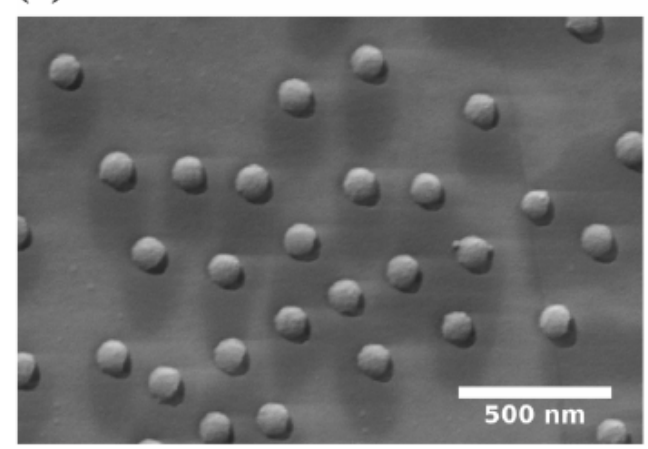

(b)

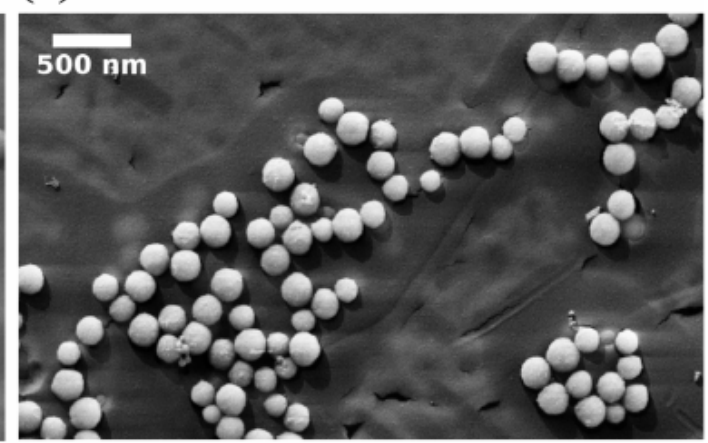

\section{Figure 7}
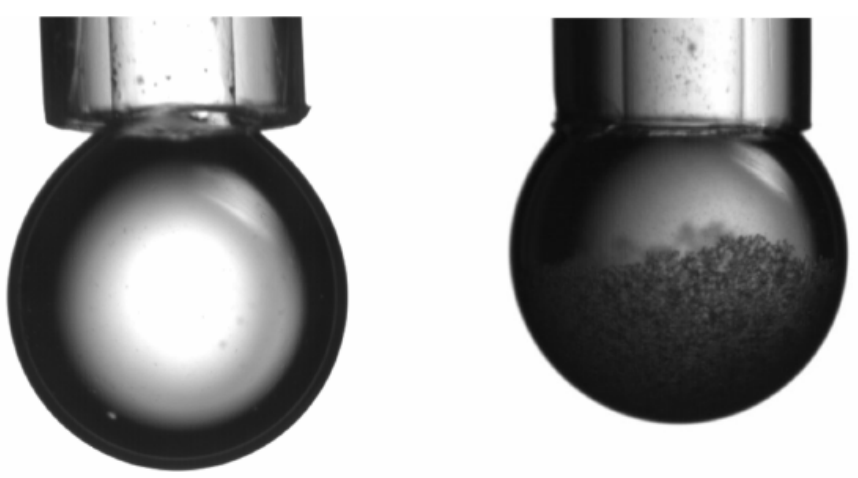
(a) Homogeneous
PMMA ( (b) Funct
nanoparticles
silica nanoparticles 


\section{Figure 8}

a

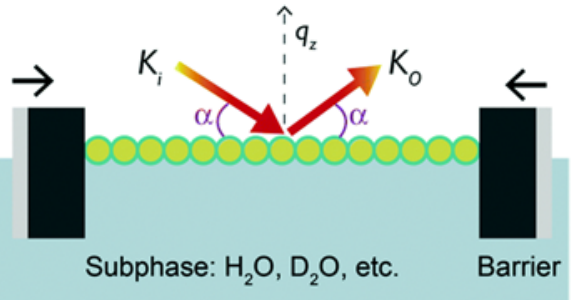

b

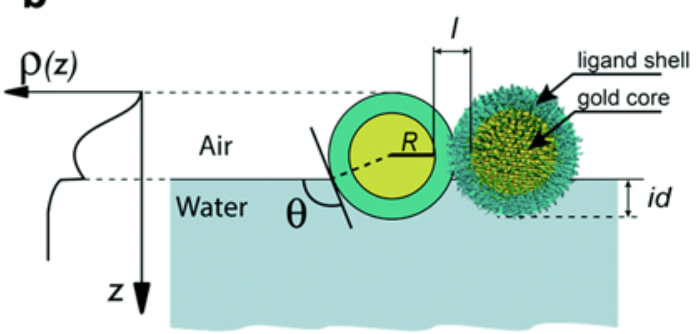

\section{Figure 9}

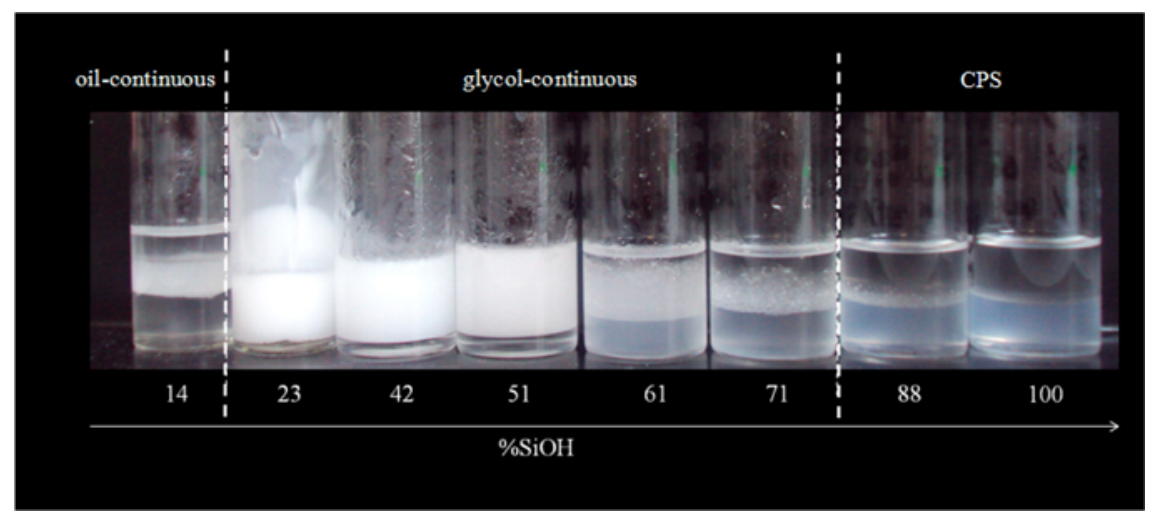


Figure 10

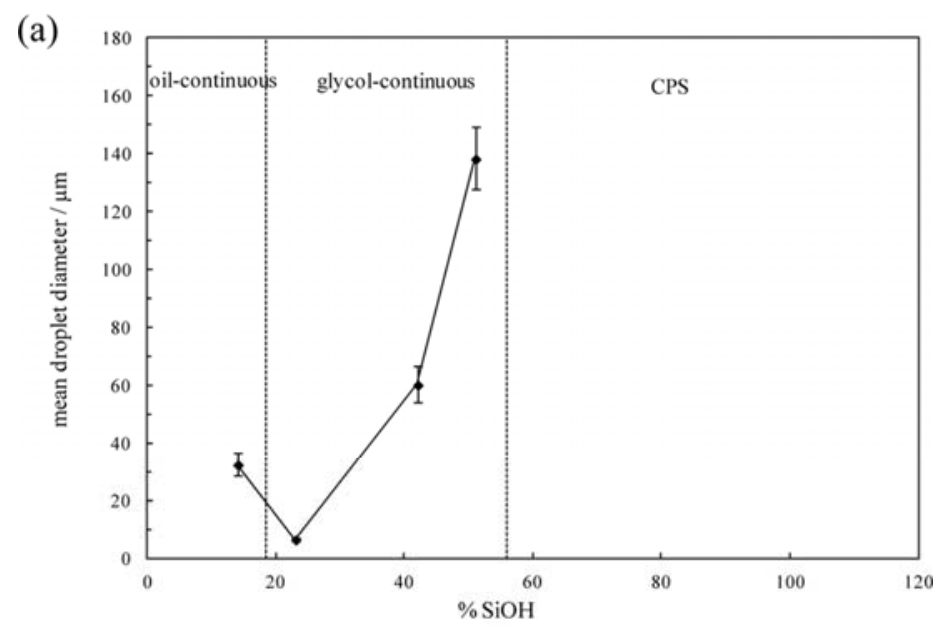

(b)
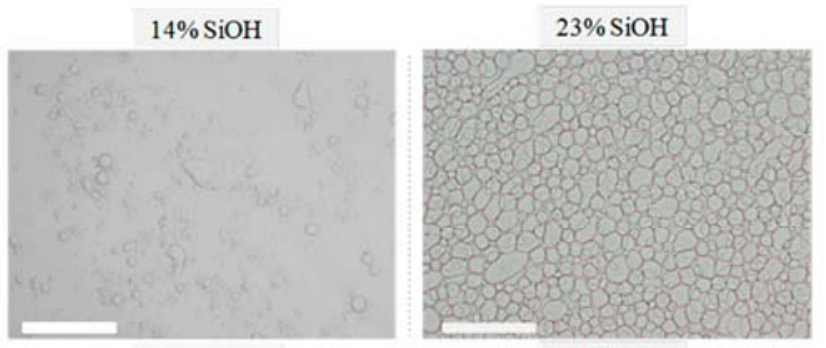

$42 \% \mathrm{SiOH}$

$51 \% \mathrm{SiOH}$
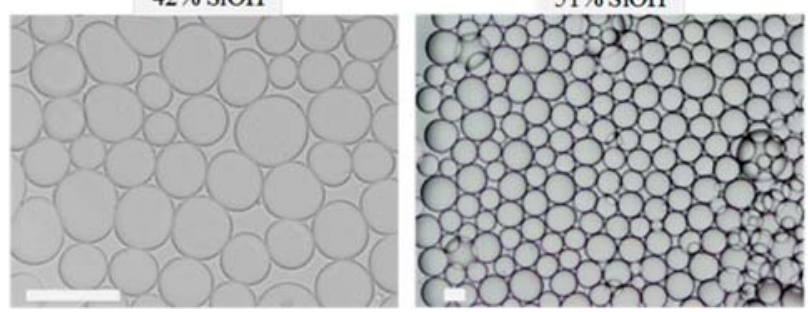
Figure 11
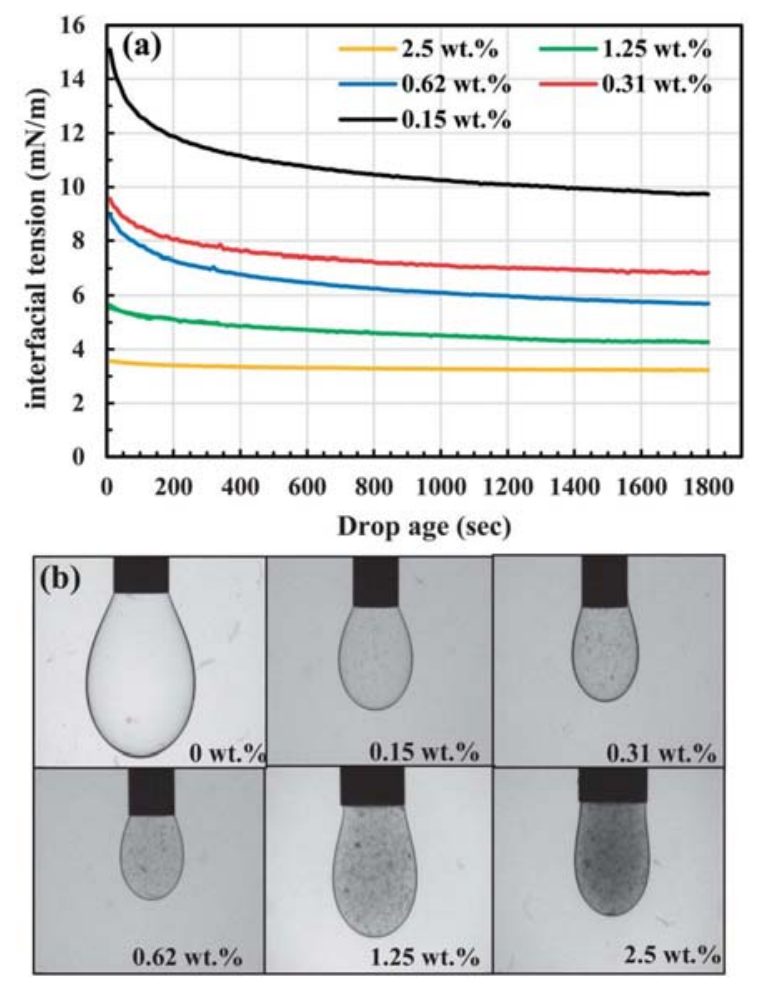


\section{Figure 12}
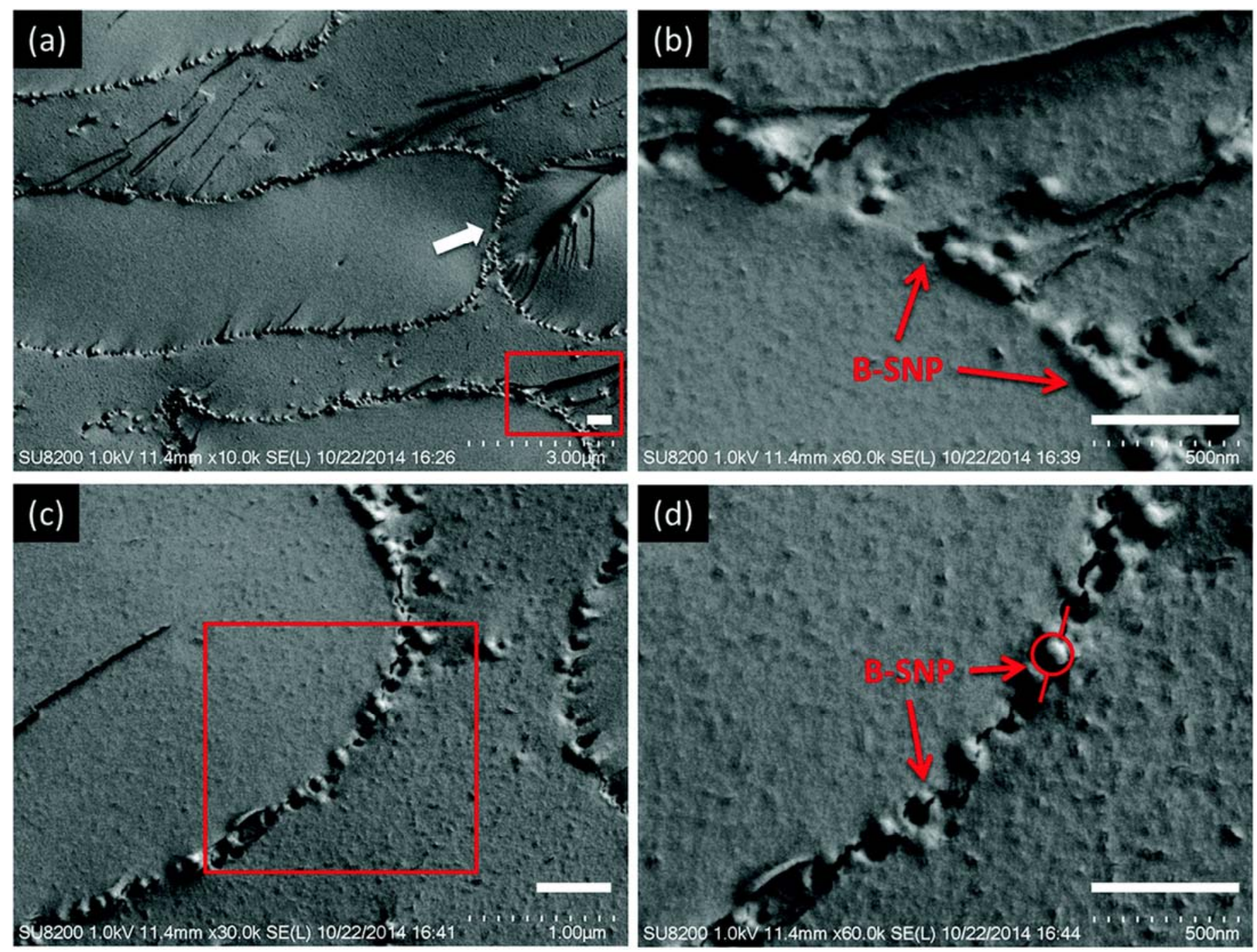
Figure 13

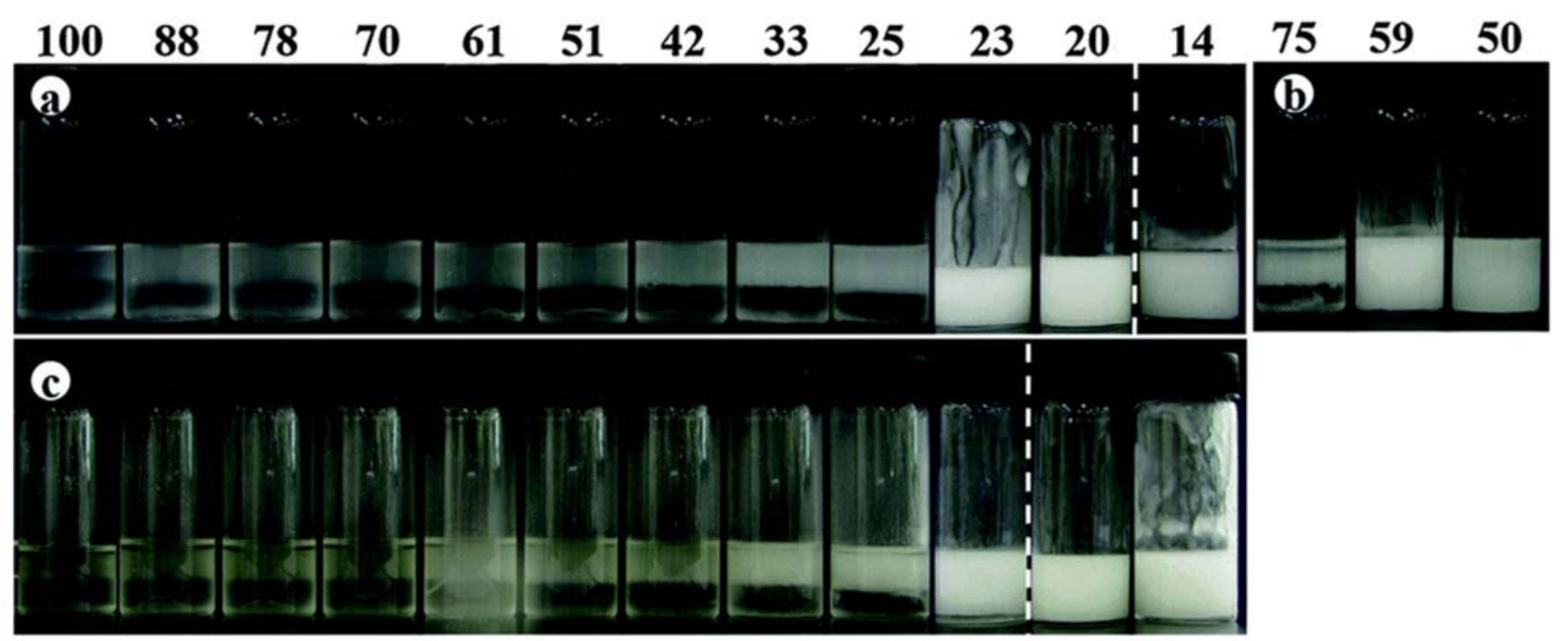


Figure 14
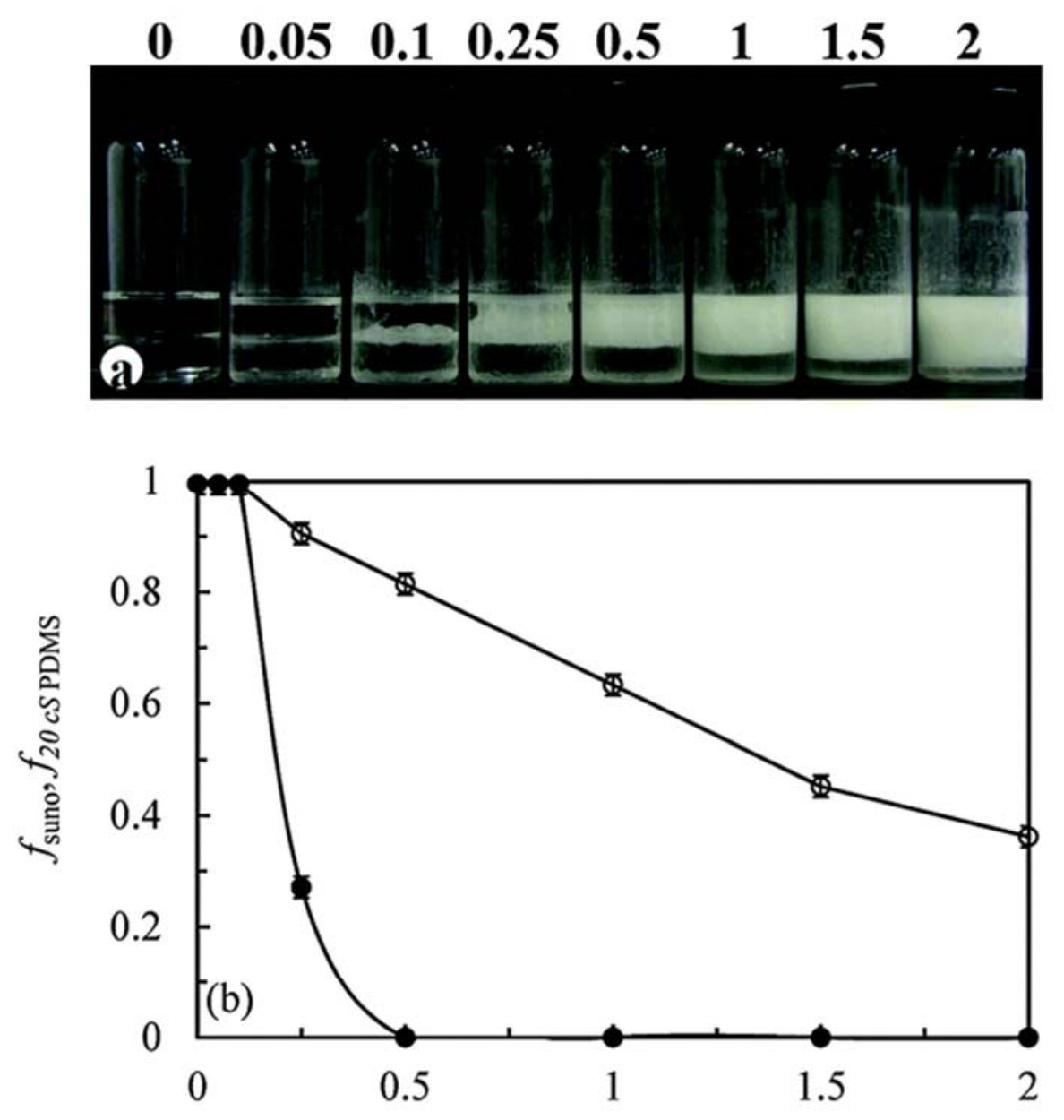

[PF-5 Eight Pearl 300S-Al particles]/wt.\%

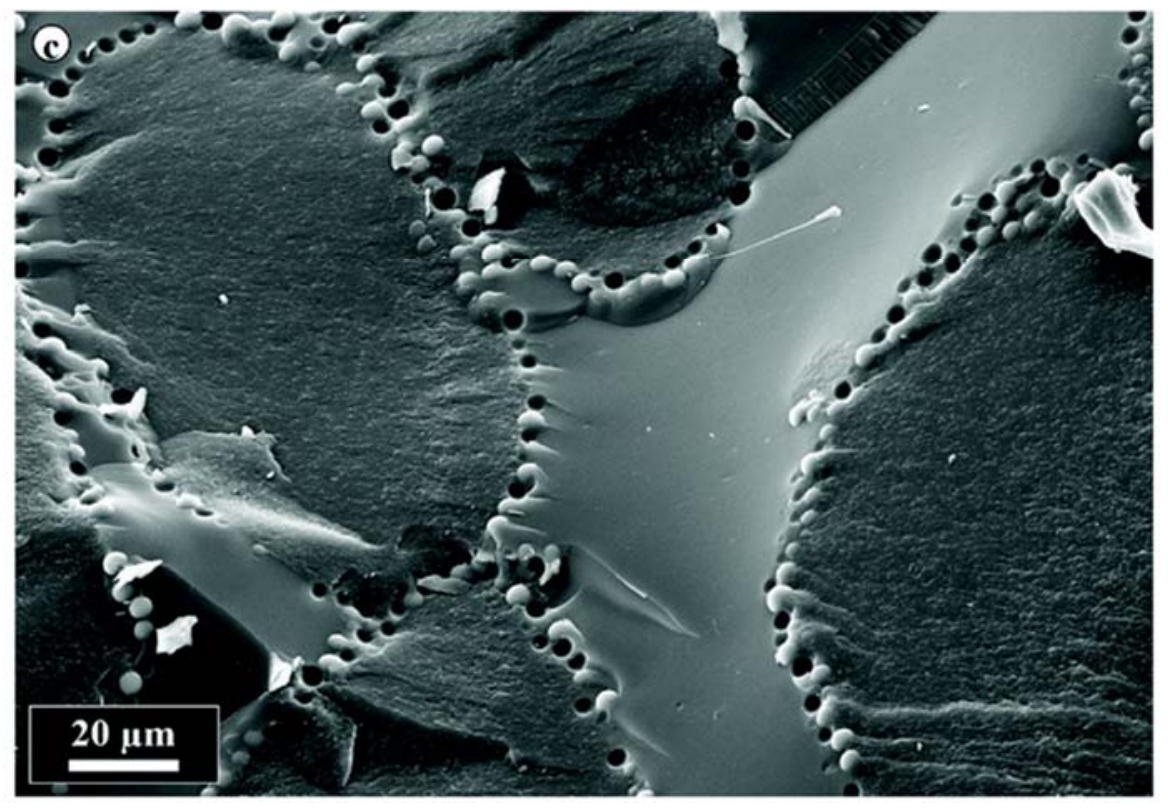


Figure 15

(a)

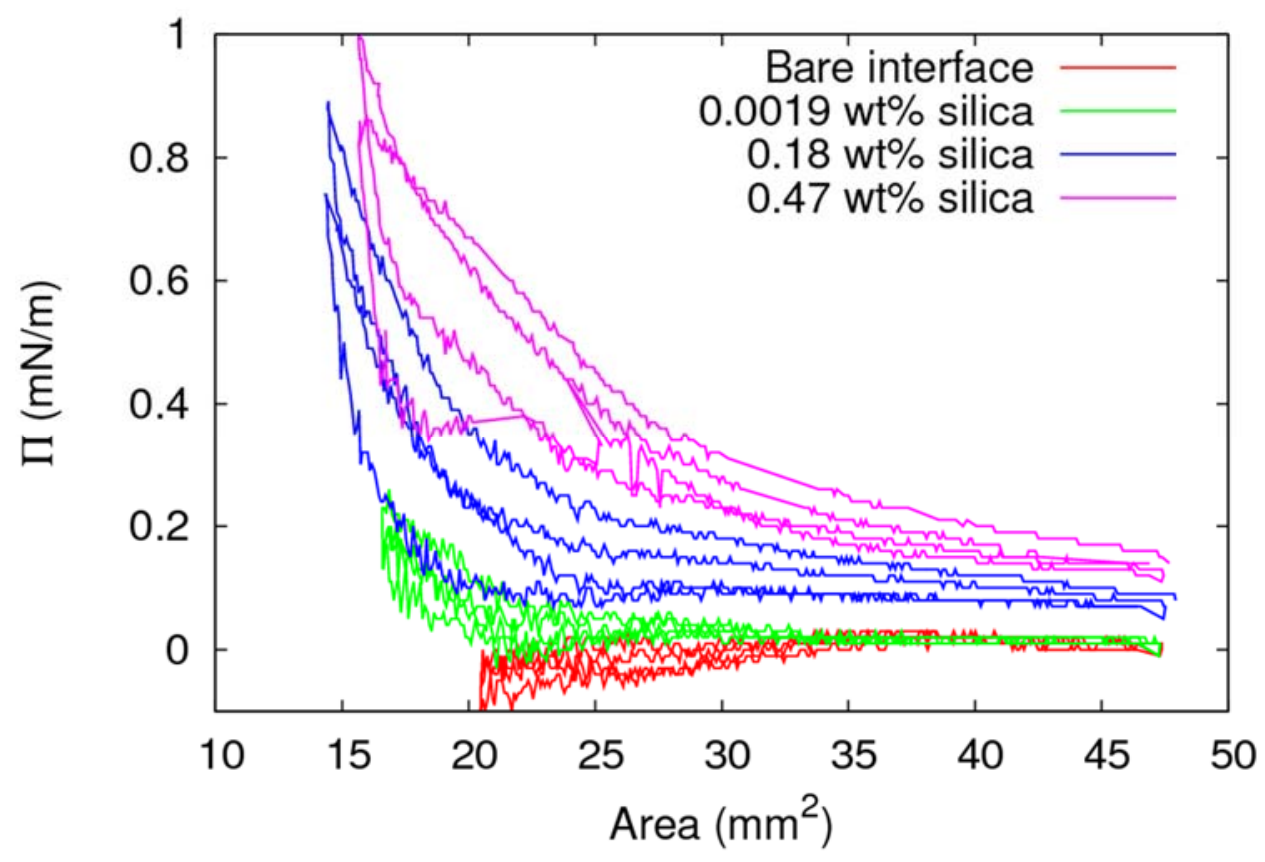

(b)

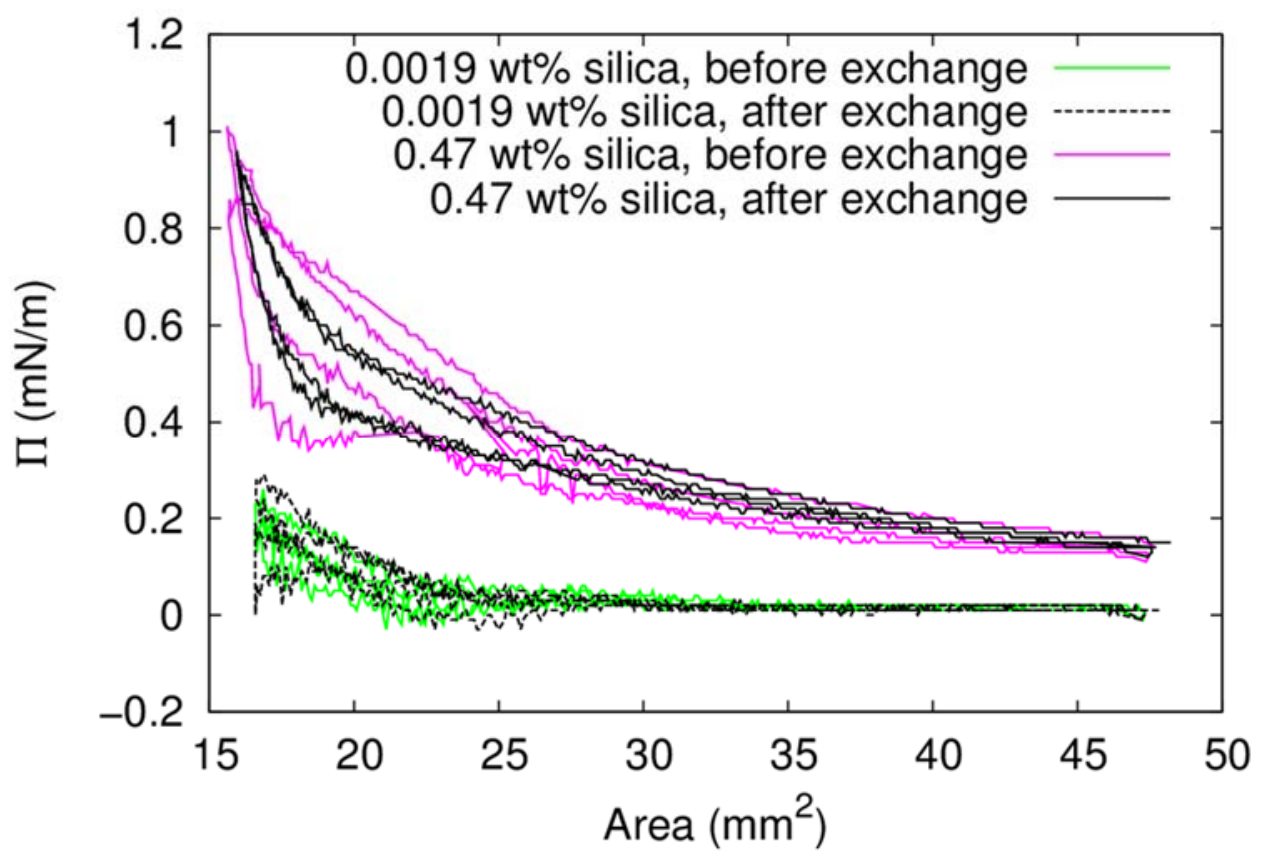


Figure 16

(a)

0.0019 wt\% silica $40 \mu \mathrm{L}$

0.0019 wt\% silica $20 \mu \mathrm{L}$

0.0019 wt $\%$ silica $5 \mu \mathrm{L}$

0.47 wt\% silica $40 \mu \mathrm{L}$

0.47 wt $\%$ silica $20 \mu \mathrm{L}$

0.47 wt $\%$ silica $5 \mu \mathrm{L}$

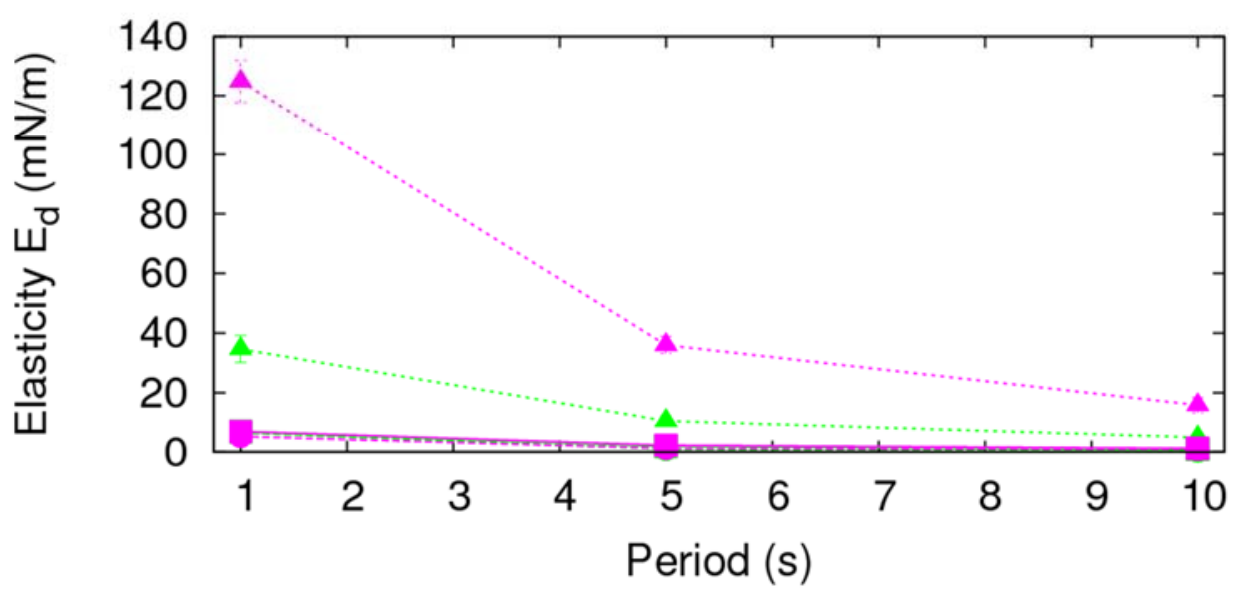

(b)

0.0019 wt $\%$ silica $40 \mu \mathrm{L}$

0.0019 wt\% silica $20 \mu \mathrm{L}$

0.0019 wt $\%$ silica $5 \mu \mathrm{L}$

0.47 wt $\%$ silica $40 \mu \mathrm{L}$

0.47 wt\% silica $20 \mu \mathrm{L}$

0.47 wt $\%$ silica $5 \mu \mathrm{L}$

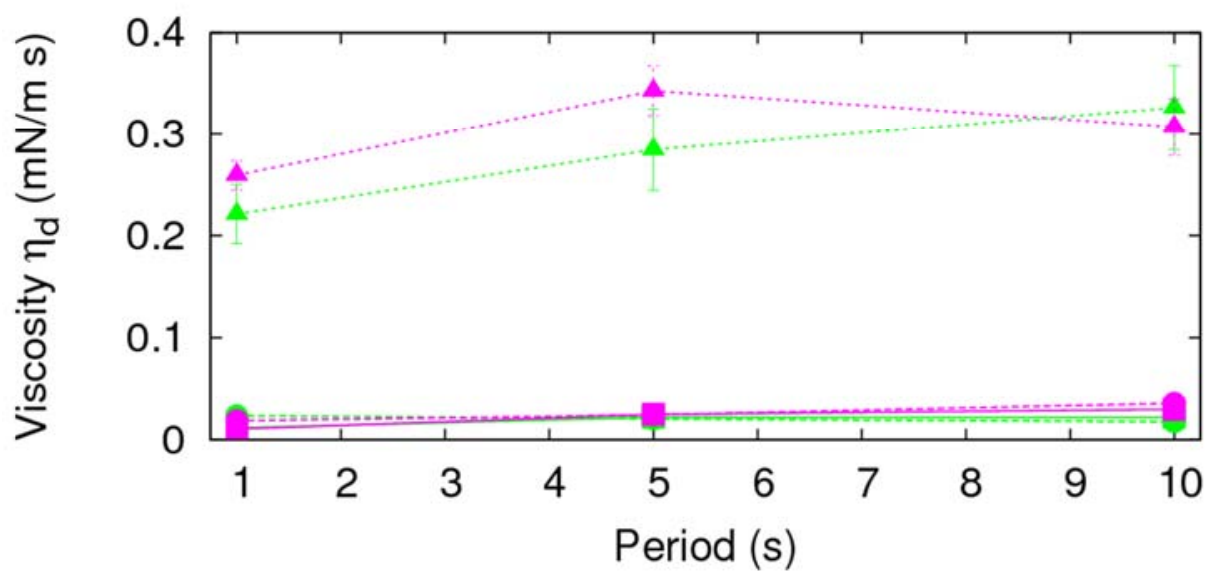


Figure 17

(a)

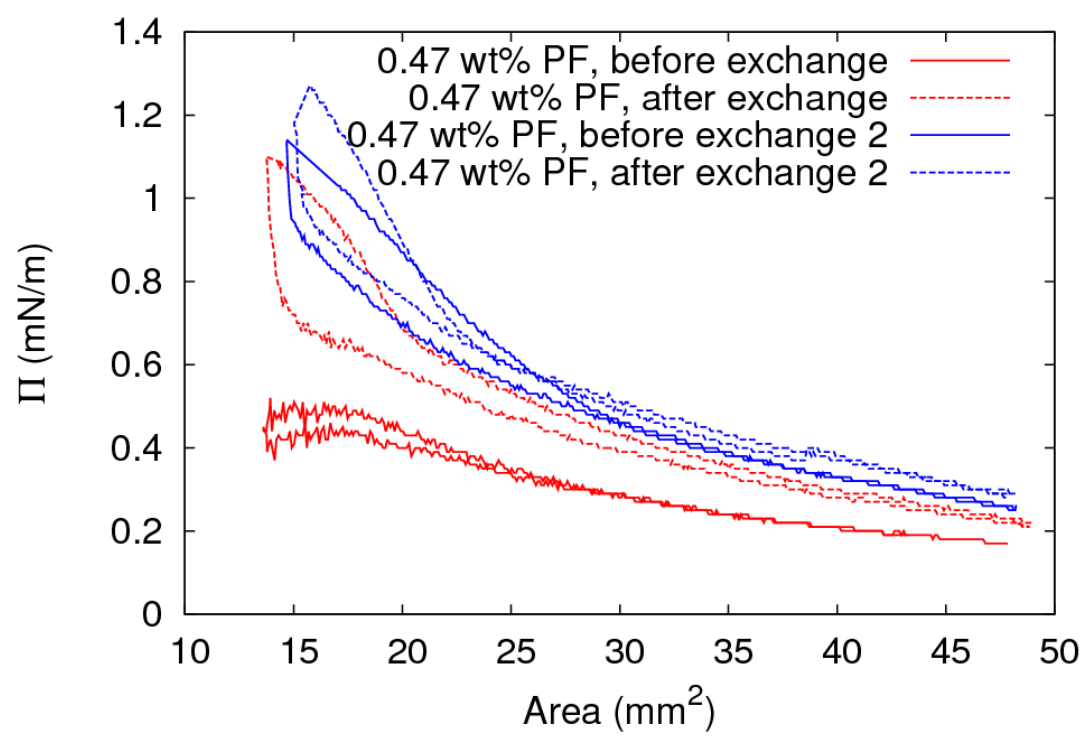

(b)

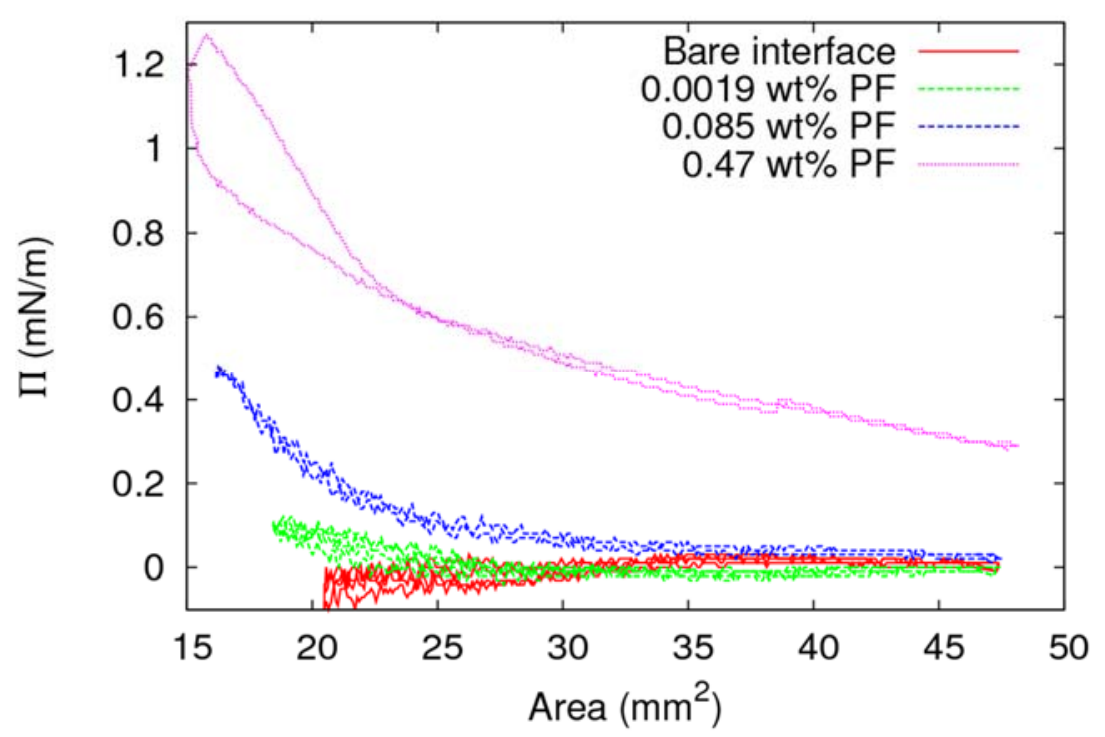

Figure 18

(a)

(b)

(c)
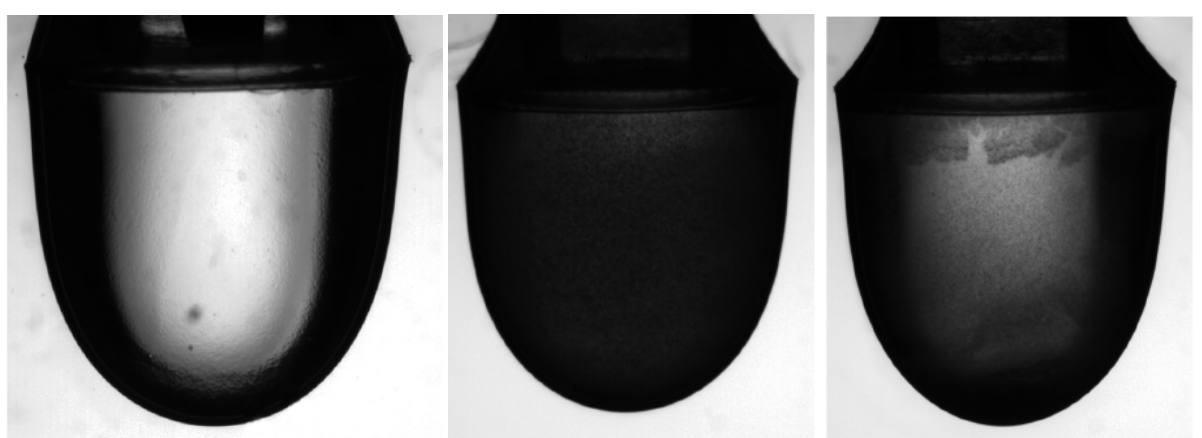